Abstract In the past two decades, semiconductor quantum dots and wires have developed into new, promising classes of materials for next-generation lighting and display systems due to their superior optical properties. In particular, exciton-exciton interactions through nonradiative energy transfer in hybrid systems of these quantum-confined structures have enabled exciting possibilities in light generation. This review focuses on the excitonics of such quantum dot and wire emitters, particularly transfer of the excitons in the complex media of the quantum dots and wires. Mastering excitonic interactions in lowdimensional systems is essential for the development of better light sources, e.g., high-efficiency, high-quality white-light generation; wide-range color tuning; and high-purity color generation. In addition, introducing plasmon coupling provides the ability to amplify emission in specially designed exciton-plasmon nanostructures and also to exceed the Förster limit in excitonic interactions. In this respect, new routes to control excitonic pathways are reviewed in this paper. The review further discusses research opportunities and challenges in the quantum dot and wire excitonics with a future outlook.

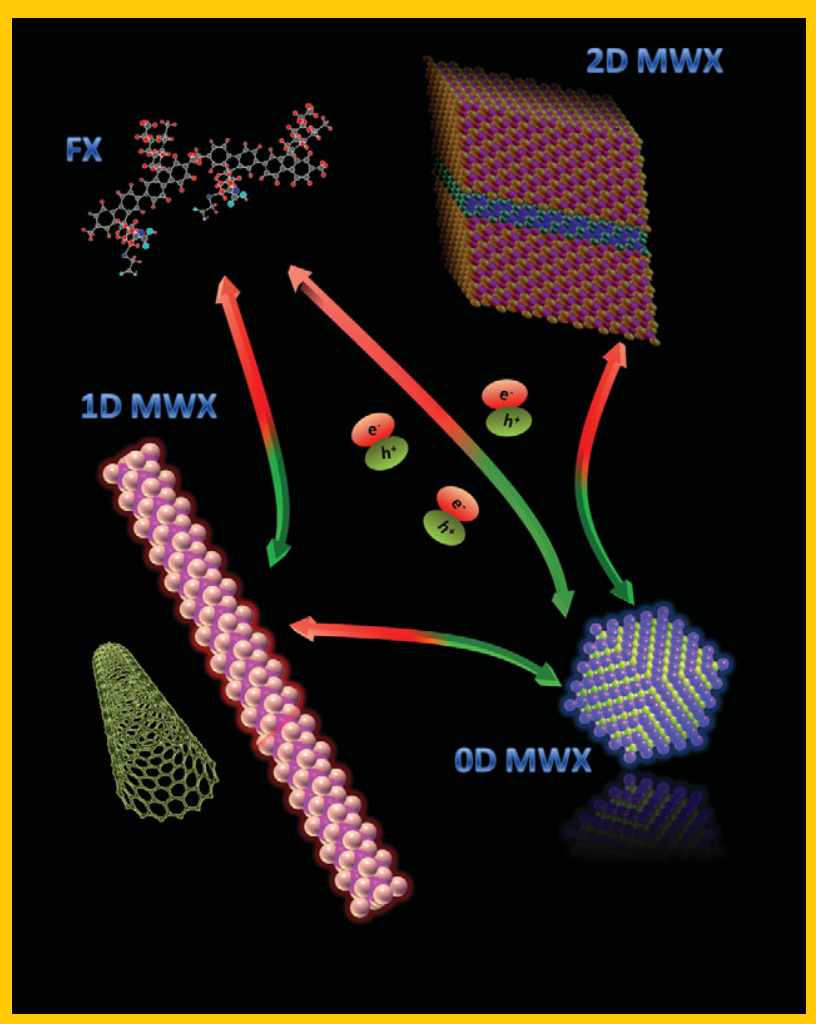

\title{
Excitonics of semiconductor quantum dots and wires for lighting and displays
}

\author{
Burak Guzelturk ${ }^{1,2}$, Pedro Ludwig Hernandez Martinez ${ }^{1,2}$, Qing Zhang1, Qihua Xiong ${ }^{1}$, \\ Handong Sun ${ }^{1}$, Xiao Wei Sun ${ }^{1}$, Alexander O. Govorov ${ }^{3}$, and Hilmi Volkan Demir ${ }^{1,2, *}$
}

\section{Introduction}

In the past two decades, the rise of quantum-confined materials has been experienced and this is anticipated to continue in the next decades. These quantum-confined materials including quantum dots and wires are capable of providing favorable and unique optical properties, which make them promising for numerous applications in photonics. These properties genuinely include excitonic features due to the strong confinement effects, which are generally not observed in their bulk counterparts. Therefore, it is essential to understand the nature of such excitonic properties in these quantum-confined materials to engineer their potential to full extent. In particular, quantum dots and wires are propitious systems for light-emitting applications given their material quality. Today, the excitonic properties in the nanoscale systems of these confined materials are carefully engineered towards highperformance solid-state lighting, displays and lasers. In this review, we bring together the essential and important works on the excitonic properties of the quantum dots and wires with a strong focus on different nanoscale systems of these confined materials suitable for lighting and displays.

\footnotetext{
${ }^{1}$ Luminous! Center of Excellence for Semiconductor Lighting and Displays, School of Electrical and Electronic Engineering, Division of Physics and Applied Physics, School of Physical and Mathematical Sciences Nanyang Technological University, Singapore 637371, Singapore

${ }^{2}$ Department of Electrical and Electronics Engineering, Department of Physics, UNAM-Institute of Materials Science and Nanotechnology, Bilkent University, Ankara 06800, Turkey

${ }^{3}$ Department of Physics and Astronomy, Ohio Univeristy, Athens, Ohio, 45701, United States

*Corresponding author: e-mail: volkan@ bilkent.edu.tr
} 


\subsection{Control of excitonic interactions}

Excitons are bound electron-hole pairs that are coupled through Coulombic interactions, or they can be considered as excitation energy packets, which exist in various material systems, including bulk semiconductors, quantum-confined inorganic nanostructures (quantum wells, wires and dots) and organics (conjugated polymers, dyes, and luminescent proteins). In recent years, the study of excitonic interactions in the nanoscale systems as the enabling means of controlling fundamental excitonic properties for light generation and harvesting is emerging as a new field, which we call excitonics here. Such excitonically engineered systems rely on the efficiency of exciton recombination and/or exciton dissociation, which are governed by the interaction among excitons, and those between excitons and other quasiparticles like photons, phonons and plasmons. The title of this review also employs the term excitonics, since the essential excitonic interactions are reviewed. Excitonically engineered materials have the potential to offer tunable optical properties; therefore, they constitute a promising class of materials for the future optoelectronic technologies for lighting, optical detection, and solar energy-harvesting purposes. Therefore, it has become crucial to develop a deeper understanding of the excitonic interactions (nonradiative energy transfer, Dexter energy transfer, exciton diffusion and dissociation, exciton-plasmon interaction) in complex media to achieve excitonically engineered systems.

\subsection{Nonradiative energy transfer}

Nonradiative energy transfer (NRET), which was first correctly described by Theodor Förster [1-3], is also known as Förster resonance energy transfer (FRET) or resonance energy transfer (RET). NRET is the transfer of the excitation energy from an excited-state molecule (donor) to a ground-state molecule (acceptor) without the process of photon emission/reabsorption. The physical mechanism behind this emissionless energy transfer is explained by the near-field Coulombic interactions between the resonant transition dipoles, which is also known as a dipole-dipole coupling. This nonradiative character of the process ensures a high efficiency of NRET.

The classical formulation of the NRET rate and efficiency has been described in detail in various textbooks and reviews $[4,5]$. A single donor-acceptor pair, which is the simplest case to study and formulate, is helpful for understanding the NRET and its involved parameters. The NRET rate, $k_{\mathrm{NRET}}$, for the case of a single donor-acceptor pair is given in Eq. (1)

$$
k_{\mathrm{NRET}}(R)=\frac{9 \kappa^{2} c^{4}}{8 \pi \tau_{\mathrm{D}} n^{4} R^{6}} \int F_{\mathrm{D}}(\omega) \sigma(\omega) \frac{\mathrm{d} \omega}{\omega^{4}},
$$

where $\kappa$ is the orientation factor that depends on the donoracceptor transition dipole arrangement; $c$ is the speed of light; $n$ is the refractive index of the energy transfer medium; $\tau_{\mathrm{D}}$ is the radiative lifetime of the donor; $R$ is the actual sep- aration between the donor and the acceptor; $F_{\mathrm{D}}(\omega)$ is the normalized fluorescence spectrum of the donor; $\sigma(\omega)$ is the absorption cross section of the acceptor; and $\omega$ is the optical frequency in radian/s. The integral term is also called the spectral overlap $(J)$ between the donor emission and the acceptor absorption, which corresponds to the strength of the resonance condition. $\tau_{\mathrm{D}}$ can be calculated using the fluorescence lifetime of the donor in the absence of the acceptor $\left(\tau_{\mathrm{ff}}\right)$ and the donor fluorescence quantum yield (QY) is calculated as $\tau_{\mathrm{D}}=\frac{\tau_{\mathrm{H}}}{Q Y}$. Furthermore, a hypothetical distance that is called the Förster radius, $R_{0}$, is defined, where the NRET efficiency becomes equal to $\frac{1}{2}$ when the separation between the donor-acceptor is exactly equal to $R_{0}$. The NRET rate can also be expressed in terms of the Förster radius and the actual separation and the donor radiative lifetime by assuming a dipole-orientation factor, $\kappa^{2}$, that averages to $\frac{2}{3}$, which is typical for isotropic emitters.

$$
k_{\mathrm{NRET}}(R)=\frac{1}{\tau_{\mathrm{fl}}}\left(\frac{R_{0}}{R}\right)^{6} .
$$

The efficiency of exciton transfer can be calculated from the measured change in the decay rate of the donor in the presence of the acceptors by comparing to the donor-only exciton decay rate (inverse of the exciton lifetime):

$$
\eta_{\mathrm{NRET}}=\frac{k_{\mathrm{NRET}}(R)}{k_{\mathrm{NRET}}(R)+\tau_{\mathrm{fl}}^{-1}} .
$$

NRET is a highly distance sensitive process due to the inverse sixth power $\left(R^{-6}\right)$ dependence of the separation distance in the case of point-to-point dipole coupling. Therefore, NRET had been first used as a nanoscale ruler [6]. However, the distance dependence could be altered for different acceptor geometries such that small-molecule acceptors or 3D confined quantum dots (QDs) are considered to be infinitesimal transition dipoles, which leads to the classical $\mathrm{R}^{-6}$ dependence. By contrast, 2D and 1D confined quantum wire (Qwire) and quantum well (QW) acceptors lead to distance dependences that vary with $\mathrm{R}^{-5}$ and $\mathrm{R}^{-4}$, respectively [7]. Essentially, quantum confinement of the acceptor changes the distance dependency of the NRET. Furthermore, different assemblies of the acceptors could also alter the distance dependence, as in the case where a 2D-like assembly of the semiconductor QDs (i.e. a monolayer of QDs on a QW donor) act as a 1D confined structure, which consequently results in the distance dependence having the form of $\mathrm{R}^{-4}$ similar to QWs $[7,8]$.

NRET has historically been exploited in various areas of biology for sensing, labeling, sensitive distance measurements and understanding of the molecular-level interactions. Recently, NRET has been shown to be useful for optoelectronic technologies towards the purpose of creating efficient lighting and solar-energy-harvesting systems. For this purpose, exciton energy transfer in the QD- and Qwire-based material systems, which have physical dimensions on the order of the Förster radius, can be employed to control the photonic properties for light-generation and light-harvesting systems. 


\subsection{Dexter energy transfer, charge transfer, exciton diffusion and dissociation}

Dexter energy transfer [9], which is also known as electron exchange energy transfer, relies on the wavefunction overlap of the electronic states between different molecules in the near field. Dexter energy transfer is a short-range energy transfer unlike FRET, which is known to be a longrange energy transfer due to the working distances that are on the order of $10 \mathrm{~nm}$. Therefore, Dexter energy transfer is only effective for donor-acceptor separations, which are typically on the order of a nm or even shorter. Furthermore, Dexter energy transfer has an exponential distance dependence as compared with the $\mathrm{R}^{-4}-\mathrm{R}^{-6}$ distance dependencies in the long-range NRET processes [10]. Finally, Dexter energy transfer can also occur between nonemissive electronic states of the materials, such as spin-forbidden triplet states, whereas it is currently widely believed that these excitons cannot be transferred via NRET because they have negligible oscillator strength [11].

Another important excitonic process is the diffusion of the excitons. The excitons can diffuse in a material via NRET in the broadened density of states of the same material, which is further called energy migration. Exciton diffusion has been widely studied for organic semiconductors in the search for suitable materials for organic solar cells that have large diffusion lengths to increase the probability of the charge separation at the donor/acceptor heterointerfaces [12]. In addition to organic materials, exciton diffusion is vital in bulk and quantum-confined semiconductor structures. Excitons can be transported in the quantum-confined materials (i.e. QWs and Qwires) or in the assemblies of the QDs, which should be well understood and controlled because defects can trap the diffusing excitons such that the emission efficiencies can be significantly reduced as a result of increased nonradiative recombination of the excitons. This picture is also valid for organic semiconductors used for organic LEDs (OLED), where the exciton diffusion is not a desired process contrary to organic solar cells (OPV). In 3D confined QDs, excitons can still diffuse via interparticle NRET in the assemblies of the QDs. This exciton diffusion is one of the reasons for the observed redshifts when QDs are casted into solid-state films in addition to substrate effects [13].

Exciton dissociation is the decomposition of the bound electron-hole pairs into free carriers. This dissociation is a crucial step for excitonic solar cells [14] (bulk heterojunction [15] and dye-sensitized [16]) because the generation of free charge carriers is required to realize the photovoltaic operation. In excitonic solar cells, dissociation of the excitons is facilitated by the interfaces that have type-II band alignments to physically break the excitons into free charges. The resistance against the breaking of the exciton in terms of energy is called the exciton binding energy. Materials with a larger exciton binding energy have more stable excitons because it is difficult to overcome this large Coulomb energy within the electron-hole pairs.
Lately, excitonic processes such as multiexciton generation (MEG), Auger recombination and exciton-exciton annihilation have been studied in the quantum-confined semiconductors. Multiexciton generation, also called carrier multiplication, is the generation of multiexcitons upon the absorption of a high-energy photon $h v \geq 2 \times E_{\text {Gap }}$. It has been shown that semiconductor QDs can be quite efficient in terms of converting higher-energy photons into multiexcitons $[17,18]$. Related to the multiexciton phenomena, Auger recombination becomes severe because excitons are spatially very close to each other. In Auger recombination, the energy of the recombining exciton is transferred to another already excited charge carrier in the material such that this charge is excited into higher-energy states (i.e. a hot carrier). This hot carrier quickly thermalizes to the respective band edge by losing its energy to the phonon vibrations; therefore, Auger recombination can significantly decrease the multiexciton operation in the quantum-confined structures [19].

\section{Need for quantum confinement to control excitonics}

In excitons, electrons and holes are bound to each other with a Coulomb energy that is called the exciton binding energy $\left(E_{\mathrm{b}}\right)$. Because the binding energy is considerably greater than thermal energy $\left(k_{\mathrm{B}} T\right)$, the excitons can remain without dissociation. Although the exciton binding energy is a material-specific energy, it can be altered via confining the electrons and holes into small dimensions, as in the case of quantum confinement. Bulk exciton binding energies are approximately $4-60 \mathrm{meV}$ for the common semiconductors (see Table 1). However, through quantum confinement, the exciton binding energies can, in principle, be made considerably larger (more than an order of magnitude) than the bulk-exciton binding energies [20]. This is why quantum confinement is the key to achieving excitonic operation in inorganic semiconductors. As the confinement becomes stronger, the binding energy further increases. Consequently, the QDs exhibit the largest exciton binding energies in comparison to the Qwires and QWs of the same material due to the strong 3D confinement. For low-binding-energy materials with poor confinements, as in the case of some of the Qwires and QWs, excitonic behavior can be observed only at low temperatures. Another important excitonic parameter is the exciton Bohr radius, which is an intrinsic material property for a given bulk semiconductor. The quantum-confinement effects become dominant if the actual physical size of the material is made smaller than the exciton Bohr radius. Two (three) dimensions of the semiconductor Qwires (QDs) can in principle be made smaller than the exciton Bohr radius such that sizetunable properties arise in these confined structures. Table 1 shows the bulk exciton Bohr radius of some of the common semiconductors. 
Table 1 Bulk exciton binding energy and exciton Bohr radius are reported for the commonly used semiconductors.

\begin{tabular}{lcr}
\hline $\begin{array}{l}\text { Semiconductor } \\
\text { compound }\end{array}$ & $\begin{array}{c}\text { Bulk exciton } \\
\text { binding energy (meV) }\end{array}$ & $\begin{array}{r}\text { Exciton Bohr } \\
\text { radius (nm) }\end{array}$ \\
\hline $\mathrm{ZnO}$ & $60.0[21]$ & $1.8[22]$ \\
$\mathrm{ZnS}$ & $40.0[23]$ & $2.5[24]$ \\
$\mathrm{ZnSe}$ & $20.4[25]$ & $4.1[26]$ \\
$\mathrm{ZnTe}$ & $13.0[27]$ & $6.7[28]$ \\
$\mathrm{CdS}$ & $29.0[29]$ & $2.9[30]$ \\
$\mathrm{CdSe}$ & $15.0[31]$ & $5.6[32]$ \\
$\mathrm{CdTe}$ & $9.0[33]$ & $7.3[34]$ \\
$\mathrm{AlN}$ & $42.5[35]$ & $1.2[36]$ \\
$\mathrm{GaN}$ & $24.1[35]$ & $2.7[35]$ \\
$\mathrm{GaAs}$ & $5.1[35]$ & $14.5[37]$ \\
$\mathrm{InN}$ & $15.2[35]$ & $8.0[38]$ \\
$\mathrm{InP}$ & $4.8[39]$ & $12.0[40]$ \\
$\mathrm{InAs}$ & $1.5[35]$ & $36.0[41]$ \\
$\mathrm{PbS}$ & $0.019[42]$ & $18.0[43]$ \\
$\mathrm{PbSe}$ & - & $47.0[43]$ \\
\hline
\end{tabular}

\section{New class of emitters: Quantum dots and quantum wires}

In the last decades, we have witnessed the rise of quantumconfined nanostructures such as QWs, Qwires and QDs, which have already started to be promoted in various applications. Among these quantum-confined structures, 3D confined QDs and 2D confined Qwires are strong candidates for photonic and lighting applications [44, 45]. Semiconductor QDs, which are crystalline nanoparticles synthesized via colloidal chemistry techniques [46], have physical dimensions on the order of several nanometers and are generally smaller than the bulk exciton-Bohr radius. Therefore, strong quantum-confinement effects arise $[47,48]$. By contrast, semiconductor Qwires are grown using bottom-up techniques via either vapor phase (chemical or physical vapor deposition) [49,50] or solution-based syntheses (colloidal or hydrothermal) [51]. These Qwires are promising due to their versatile electrical and optical properties. Qwires can be quite long, on the order of micrometers, but they have small radii ranging from few nanometers to tens of nanometers; therefore, quantum-confinement effects can also be observed in the radial direction.

For light-generation and light-harvesting systems, QDs and Qwires show good prospects to replace the current materials. To date, semiconductor QDs have already been utilized as building blocks for various light-generation and light-harvesting devices [52-54]. QD-based LEDs represent an important class of LEDs that have superior performance in the state-of-the-art devices for white-light generation [55]. Likewise, Qwires have begun to emerge as auspicious materials for LEDs, lasers and solar-energyharvesting systems [56-58]. Moreover, Qwire-based LEDs, sometimes called nanoLEDs, have been shown to be efficient light sources with tunable polarization and good outcoupling properties thanks to the Qwires; therefore, they become favorable not only for solid-state lighting but also for nanoscale high-speed telecommunication and computing applications in the future $[59,60]$. The photonics properties of the QD and Qwire structures are excitonic in nature; therefore, understanding and being able to engineer the excitonic processes are of considerable importance for developing advanced and efficient optoelectronic systems based on these materials.

\section{Excitonic control in the nanocomposites of quantum dots and wires}

\subsection{Quantum dot excitonics}

QDs exhibit tunable emission spectra, high photoluminescence (PL) QY, broad absorption spectra and increased environmental stability. These properties have generated significant attraction for the use of QD devices for light generation. To date, these QDs have been utilized in light-emitting diodes (LEDs) through two primary excitation schemes: color-conversion type [91] LEDs using QDs as photoluminescent materials and electrically driven [53] type LEDs using QDs via charge injection for electroluminescence. In these devices, QDs are integrated into different material systems to create a synergy via utilizing the advantages of the constituent materials, including other QDs, QWs, Qwires, carbon nanotubes (CNTs) and organic semiconductors. Here, we will review the excitonic processes in the various composites of the QDs.

\subsubsection{QD-QD excitonic interactions}

In colloidally synthesized semiconductor QD samples, there is always a finite size distribution, which inhomogeneously broadens the emission and absorption spectra of the QDs. Consequently, excitonic interactions arise in the distribution of the same QDs, which are referred to here as homoexcitonic interactions. The homoexcitonic interactions are important to understand the optical properties of the QD samples. Additionally, heteroexcitonic interactions, which can occur between different types, sizes and compositions of the QDs, are crucial towards engineering the excitonic operation in the QD composites.

Before discussing the homo- and heteroexcitonic interactions in the QD assemblies, it is worth discussing the effects of the different media (i.e. solution-phase or solidstate films) on the optical properties of QDs. In the solution phase, QDs are more isolated from each other, unless the solution is very dense or the QDs are chemically attracted to each other; therefore, the excitonic interactions between QDs are generally negligible in solution phase. However, when cast into the solid-state in the form of close-packed films, QDs come into intimate contact with each other, 
and they consequently exhibit complex excitonic properties. Distinct differences between solution-phase and solidstate films are that the photoluminescence emission is redshifted and the PL QY is reduced in solid-state films as compared with solution-phase films. The redshift in emission spectra involves both electromagnetic field effects on the transition dipoles in solid-state films due to the substrate and increased excitonic interactions (homoexciton interactions in the same QD batch) among the QDs in the form of exciton migration towards smaller energy gap (larger size) QDs. First, the substrate leads to a change in the dielectric medium around the QDs, which causes changes in the spontaneous decay rate and energy of the transition dipole, which is a well-known phenomenon and not limited to the QDs. Consequently, the radiative lifetime is shortened and the energy of the transition dipole is decreased, which leads to the redshift [61]. Secondly, the existence of the size-distribution causes homo-NRET from smaller to larger QDs in the ensemble such that the exciton population in the QDs that are on the red tail of the spectrum. The reduction in PL-QY is attributed to the increased nonradiative recombination channels in solid-state phase. The surfaces of the QD are passivated with organic ligands. In the solution phase, these ligands can function properly such that surface traps are effectively passivated. However, in solid-state films, the stacking of the QDs may lead to improper passivation of the surfaces, which causes increased nonradiative decay channels and traps for the excitons.

The homoexcitonic effects have been shown to be important in the exciton migration in solid-state phase. For example, in the solid-state films of the highly confined silicon QDs long-range exciton transport was enabled through NRET [62]. When smaller Si QDs were utilized, a longer transport was observed due to the higher NRET rates because small QDs can facilitate efficient NRET due to their sizes that are smaller than the Förster radius. The excitons hop between different QDs multiple times until they become trapped in a large size QD surrounded with smaller QDs (i.e. larger bandgap) [63]. Accordingly, it was reported that in the QD ensembles, smaller QD lifetimes become shortened due to the exciton transfer to the larger QDs, whose lifetimes are increased due to the exciton feeding effect [63-66]. Recently, CdSe/CdS-based QDs were investigated in terms of their homoexcitonic interactions as a function of the CdS shell thickness. It was found that the homoexciton transfer in solid-state films is effectively suppressed because of the very thick CdS shells (16 monolayers, called giant QDs) [67]. As shown in Fig. 1, the emission decay curves of the QD films exhibit large differences at the high-energy tail, peak and low-energy tail of the emission spectrum, which indicates the occurrence of a homoexciton transfer at the thin CdS shell but suppression of the homo-NRET in giant shells. As a result, the decay curves measured at different spectral positions of the giant-QDs become indistinguishable.

The heteroexcitonic interactions in the QD-QD structures were investigated for QDs in a wide variety of types, sizes and compositions. Rogach et al. reviewed some of
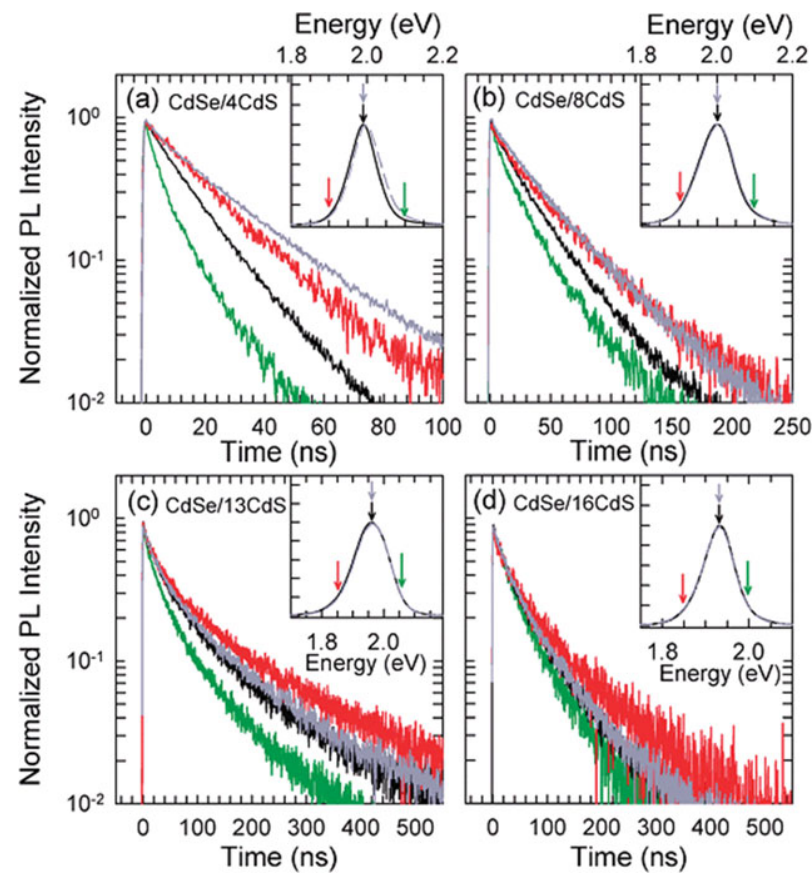

Figure 1 Time-resolved fluorescence decay measurements of the CdSe/CdS QDs depicted with respect to different CdS shell thicknesses (i.e. 4, 8, 13 and 16 monolayers). Decay measurements are performed for one QD distribution having only inhomogeneous broadening due to finite size distribution. Measurements were performed at three different spectral positions of the QD emission (i.e. higher- and lower-energy tails and at the peak) in thin film (green, black and red curves), and also at the peak position in the solution phase of the same QDs (gray curve). As the shell thickness is increased, NRET process is suggested to be suppressed in the solid-state films of the QDs because the decay curves at different positions of the QD emission spectrum become similar. Furthermore, as the shell thickness increases, the thin film and solution phase decay curves for the peak position become almost the same, which indicates the isolation of the emitting cores of the QDs due to the thick shells. [Reprinted (adapted) with permission from ref. [67] (Copyright 2012 American Chemical Society).]

the QD-based NRET structures [68]. An exciton in a QD can be transferred to another QD if the donor QD emission spectrally overlaps with the acceptor QD absorption. The transferred exciton rapidly thermalizes to the band edge (on the order of ps) in the acceptor such that back-energy transfer is not possible, unless the transfer is coherent due to strong coupling, which is typically not the case for the QD systems. Therefore, excitons have the tendency to migrate towards smaller bandgap QDs in the heterostructures. The architecture of the heterostructure plays a crucial role in the emerging exciton dynamics. To date, different QDQD-based structures have been studied in solid-state films using alternative deposition techniques, including layer-bylayer (LbL) [69,70], Langmuir-Blodgett [71], spin coating [67], drop casting [63], and blending in the polymeric host matrix [72]. 



Figure 2 Two different QD structures are described: (a) Noncascaded reference (REF) structure and (b) cascaded energy transfer (CET) structure. (b) The CET structure consists of graded layer-by-layer assembled green/yellow/orange/red/orange/yellow/green QDs. (a) The RET structure consists of layered red QDs. On the left, electronic energy levels of the graded QD-employing CET structure and the only red-emitting QD-employing REF structure are shown. On the right, steady-state PL emission is depicted for the both structures. The CET structure exhibits substantial enhancement in the PL emission as compared with the REF structure due to the trapped exciton recycling effect. [Reprinted (adapted) with permission from ref. [69] (Copyright 2004 American Chemical Society).]

Utilization of layer-by-layer QD films with graded bandgap energy was exploited as a means of enhancing the light generation in QDs. This enhancement depends on the recycling of the trapped excitons [69,70]. Figure 2 shows the designed cascaded energy transfer (CET) structure, which is composed of graded-bandgap LbL-deposited QDs, and a noncascaded reference structure (REF) that consists of only red-emitting QDs. In the CET structure, the steady-state PL emission was considerably increased as compared with the REF sample. This enhancement is attributed to the fact that the excitons, which were trapped in the subbandgap states of the QDs, can be transferred to smaller energy gap QDs. This recycling of the trapped excitons leads to a substantial increase in the PL emission of the acceptor QD. This scheme has been applied to colorconversion-based LEDs of QDs to enhance the conversion efficiency of the pump photons [72-75].

The rate of exciton transfer in the QD structures has been the subject of several studies $[63,71,73]$. Because of the size distribution of the QD samples, high NRET rates cannot be ensured in random assemblies of the QDs. However, NRET rates as high as $50 \mathrm{ps}^{-1}$ with $80 \%$ efficiency were obtained using CdTe QDs with a narrow size distribution in LbL-assembled samples [73]. In addition to intrinsic QD properties, organic ligands, which are in charge of passivating the QD surfaces, have also been shown to affect the exciton transfer. Ligands have been shown to change the nature of the transition dipole in the QD such that higherorder multipoles should be considered to account for the observed NRET in the QD-QD ensembles [62]. Furthermore, the capability of ligands to passivate the defect and trap sites on the surfaces directly influence the competing exciton transfer rate because exciton decay pathways can be altered via extra nonradiative channels of the surface defects [76].
NRET between QDs has also been investigated from the theoretical point of view [77-80]. Förster resonance energy transfer is accounted primarily for the observed exciton transfer in the ensembles of the QDs due to the effects of the polydispersity and inhomogeneous broadening [77]. However, NRET between single QDs could not be well described with classical FRET. In the case of molecular emitters such as dyes in the process of NRET, the resonance condition is satisfied by the existence of the spectral overlap between the donor emission and acceptor absorption. This resonance condition was also discussed in the NRET process for the QD-QD assemblies and was shown that totally resonant or slightly resonant electronic states could perform NRET through direct or phonon-assisted transfer of the excitons [78]. Later, two studies questioned the validity of the dipole-dipole coupling approximation for QD structures, and it was shown that the dipole-approximation is valid for donor-acceptor separation distances that are considerably greater than the molecular dimensions [81, 82] therefore, the FRET approach generally provides results that are compatible with the experimental observations.

\subsubsection{QD-QW excitonic interactions}

Epitaxially grown QWs have importance for various optoelectronic devices, and they have already become the building blocks for various optoelectronic devices, such as LEDs, lasers, photodetectors, light modulators and photovoltaic devices [83]. The current state-of-the-art inorganic LEDs are based on epitaxially grown QWs. These LEDs can be made very efficient, yet it is not easy to tune the emission color for the generation of white light. The common route to overcome this problem is the utilization of 
the color-conversion technique, which relies on a pump LED and color-converting phosphors. Multiple phosphors (green, yellow and red) are utilized on top of blue-emitting QW-LEDs to realize the color conversion. However, these phosphors are limited by their optical properties, such as their broad emission spectra that extend into the far red region in the case of red phosphors, which is spectrally out of the sensitivity of the human eye. By contrast, semiconductor QDs exhibit superior optical properties, including a very narrow full width at half-maximum (FWHM) and tunable emission spectrum in the visible range [55]. Therefore, various QD-QW systems have been proposed as efficient colorconversion materials [84-90] and have recently been reviewed [55]. These QD-integrated color-conversion LEDs only utilize the radiative energy transfer from the QWs to the QDs. Although high-quality white-light generation has been shown to be feasible, radiative-energy-transfer-based QW-QD color-conversion systems have some limitations. First, there is a loss mechanism of the pump photons due to the light outcoupling from the high refractive index pump LED into the QD-deposited color conversion layer (generally, QDs are encapsulated in a glass-like silicone resin that has a low refractive index). The other limitation is that the nonradiative recombination channels in the pump LED restrict the efficiency of the pumps' photon usage. To overcome these problems, Achermann et al. experimentally demonstrated an alternative approach, which is pumping the QDs by QW excitons through NRET in the QW-QD architectures [91]. This type of exciton pumping was first proposed by Basko et al. for QW-organic emitter system [92]. The proposed exciton pumping of the QDs involve transfer of the excitation energy from a QW to a QD that is in close proximity. To achieve the NRET pumping of the QDs, an InGaN/GaN-based multi-QW system was used as a working pump LED platform. A GaN capping layer, which is used to passivate the QWs and provide electrical contacts, was thinned to a few nanometers to have an average donor (QW)-acceptor (QD) separation on the order of the Förster radius. With this excitonic pumping of the
QDs, the light-outcoupling problem is surmounted because the pump photons are not needed to be emitted into the far field, but are transferred in the near-field via dipole-dipole coupling. Additionally, NRET creates a competing channel against the traps and the defects in the QWs such that some of the excitation energy, which was otherwise wasted, could be recycled by transferring them to the QD acceptors. Using this NRET pumping scheme, it was shown that the color-conversion efficiency can be boosted even utilizing a single monolayer of CdSe QDs on top of InGaN/GaN QWs capped with $3 \mathrm{~nm}$ of GaN. The color-conversion efficiency for this monolayer QD conversion layer was reported to be as high as $13 \%$ [93]. Later, several groups demonstrated that NRET-facilitated pumping is not limited by only QD acceptors but organic emitters, such as conjugated polymers, can also be employed as efficient acceptors [94-99]. A similar scheme was even applied to light-harvesting systems by transferring the excitons from QDs to QWs [100-102]. Nevertheless, initial demonstrations of the exciton-pumped QD-QW-based color-conversion LED structures were limited in terms of the NRET rates and efficiencies because of the limited interaction volume between the QDs and the QWs. Although the GaN capping layer could be thinned to make the QWs and QDs closer, the resulting NRET was still restricted because only the top QW and the bottom QD layer could effectively interact. For the other QD and QW layers, NRET was not expected to be efficient due to separation distances greater than $10 \mathrm{~nm}$.

Several groups proposed and demonstrated nanostructured pump LED architectures to promote the NRET between QWs and QDs as compared with the NRET in the geometrically limited planar architectures [103-105]. These nanostructured pump LED architectures generally employ top-down fabricated nanopillars or nanoholes of the InGaN/GaN multi-QWs. Nizamoglu and coworkers reported a nanopillar architecture of InGaN/GaN QWs, which is intimately integrated with $\mathrm{CdSe} / \mathrm{ZnS}$ QDs, resulting in NRET efficiencies up to $83 \%$ for red, $80 \%$ for orange and $79 \%$ for yellow-emitting QD acceptors [104, 106]. Figure 3

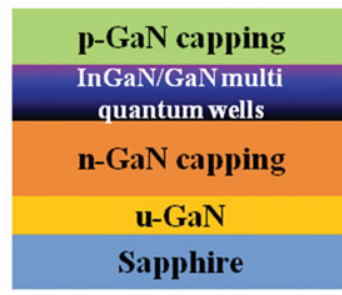

a

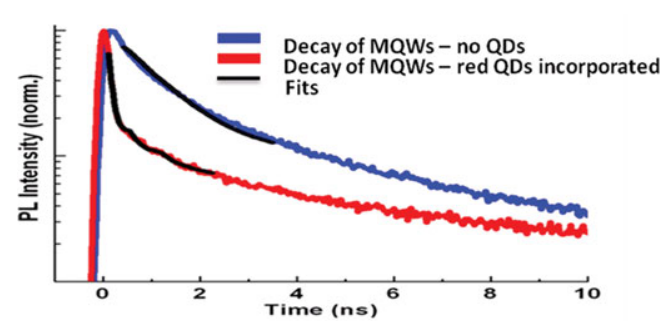

b
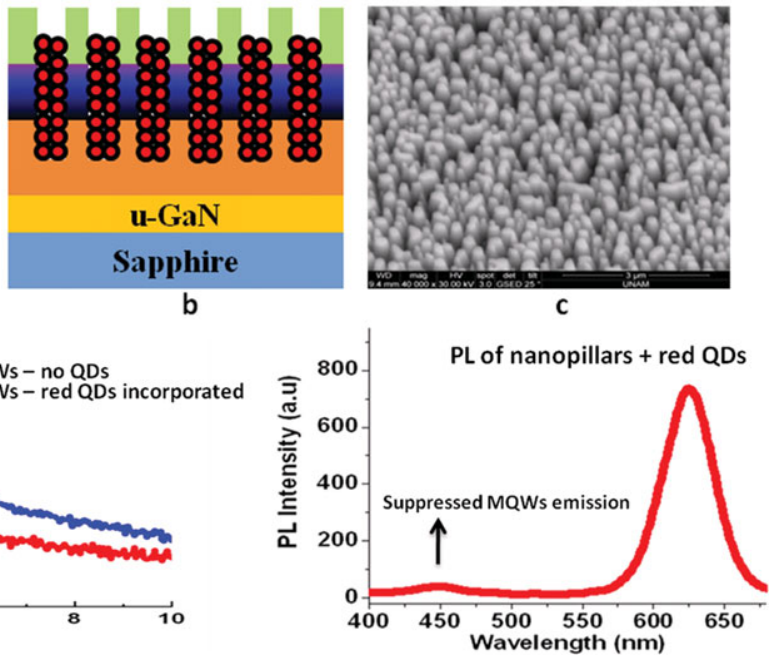

Figure 3 (Top) Schematic illustration of the InGaN/GaN multi-QW architecture and the QD-integrated hybrid. The scanning electron micrograph of the fabricated nanopillar structure is also shown. (Bottom) Time-resolved and steady-state PL spectra of the hybrid structure. In the time-resolved PL, exciton decay in the $\mathrm{QW}$ is measured before and after the incorporation of the QD. The steady-state PL measurement indicates that the $\mathrm{QW}$ emission is almost quenched due to the efficient NRET. [Reprinted (adapted) with permission from ref. [106] (Copyright 2012 Optical Society of America).] 
presents a schematic of the nanostructured (i.e. nanopillar) QW architecture with integrated QDs. A scanning electron microscopy image of the top-down fabricated InGaN/GaN nanopillars, which enable a large interaction volume between the donor and acceptor, is also shown in this figure. Furthermore, all the multi-QWs in the pump LED can now contribute to the pumping of the QDs because the QDs completely surround the nanopillars. In Figure 3 (bottom), time-resolved and steady-state PL measurements of the QW-QD structure are presented. The exciton decay of the QWs becomes faster upon incorporation with the QDs, which indicates that an efficient NRET channel has been created. From the steady-state PL spectrum of the hybrid QD-QW structure, almost totally quenched emission of the QWs can be observed upon introduction of a thin QD layer (several monolayers) on the nanopillar structure.

Recently, exciton pumping in the LbL-deposited graded energy gap CdTe QDs on planar InGaN/GaN QWs were investigated and compared with a nongraded QD acceptor layer. The graded bilayer of the CdTe QDs that consisted of green- and red-emitting QDs (QW-green QD-red QD) exhibited enhanced exciton pumping into the top red QDs (NRET efficiency of $83.3 \%$ ) as compared with the reference sample of a bilayer of red-emitting QDs exhibiting much lower NRET efficiency of $50.7 \%$ [107]. The underlying reason was explained via theoretical modeling of the exciton population evolution in the near-field. The gradient structure enabled faster and unidirectional transfer of the excitons from the QWs into the red-emitting QDs via channeling through the green QDs. In the case of the control sample, the back-and-forth NRET was theoretically shown to slow the exciton flow from the QW into QDs.

\subsubsection{QD-Qwire excitonic interactions}

QDs integrated into Qwires were demonstrated and investigated for optoelectronics with more emphasis on lightharvesting applications due to the synergistic combination of the strong light-absorption properties of the QDs and the superior electrical transport properties of the Qwires. QDs have limited electrical transport properties due to their organic ligands acting as barriers for the carrier transport. Thus, highly conductive and confined Qwires have great interest as potential hybrid systems when combined with QDs for photovoltaics and photodetectors. Kotov and coworkers investigated semiconductor CdTe Qwires as exciton acceptors, where the colloidal CdTe QDs function as strong light absorber and exciton donor in the specifically functionalized hybrid structure, as shown in the inset of Figure 4 [108]. As the QDs are integrated into the Qwires, their PL emission spectrum exhibited changes, which are explained by the exciton transfer from the QDs into the Qwires. To further enhance the sensitization of the CdTe Qwires, a cascaded energy system, which consists of green- and orangeemitting CdTe QDs, was utilized. The excitons were efficiently funnelled to the Qwires via a two-step NRET process. Later, Madhukar and coworkers demonstrated a QD-Qwire light-harvesting system and verified that the sensitization of the Qwires principally occurs via NRET, which was understood through time-resolved photocurrent spectroscopy $[109,110]$. Dorn et al. proposed and investigated $\mathrm{CdSe} / \mathrm{CdS}$ QDs integrated into CdSe Qwires as an efficient exciton-harvesting platform [111]. Furthermore, Hernandez-Martinez and Govorov investigated the NRET dynamics between QD donors and Qwire acceptors with a theoretical model and revealed that quantum confinement of the acceptor Qwire alters the distance dependence to be $\mathrm{R}^{-5}$ [112].

The use of QD-Qwire hybrids towards light generation was also investigated. The transfer of the Qwire excitons into QDs has been realized, especially for $\mathrm{ZnO}$-based Qwires, which can pump the QDs excitonically through NRET [113-115]. ZnO is one of the most suitable materials for this type of excitonic operation due to its very large exciton binding energy. However, in addition to the proofof-concept demonstration of the exciton transfer from $\mathrm{ZnO}$ Qwires to semiconductor QDs, the full potential of the

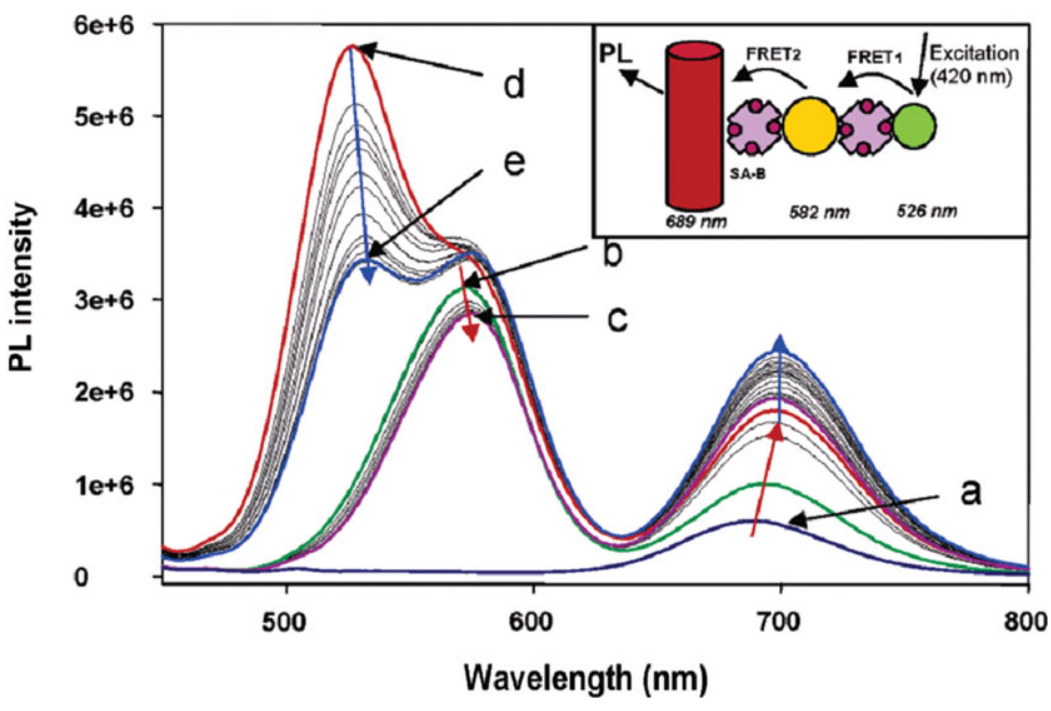

Figure 4 Exciton energy transfer sensitization of the CdTe Qwires by CdTe QDs of two different sizes (orange- and green-emitting) that are specifically attached to the Qwires with an energy gradient structure (Qwire-orange QDgreen QD). Steady-state PL spectra are shown for different cases, which indicate that the emission of the QDs is quenched but the emission of the Qwire is enhanced due to the exciton funneling. These systems are promising for excitonically enabled light-harvesting systems. [Reprinted (adapted) with permission from ref. [108] (Copyright 2005 American Chemical Society).] 
exciton pumping via $2 \mathrm{D}$ confined structures should be investigated and compared to QW-QD-based schemes.

Another important class of 2D confined Qwire structures are carbon nanotubes (CNTs). The excitonic nature of CNTs will be discussed in the section on Qwire excitonic interactions. Here, we will describe the QD-CNTbased nanostructures and the underlying excitonic operation. The composite structures of the QDs and CNTs have been characterized by several groups, and two reviews highlight the possible schemes of creating hybrid composites of the QDs and the CNTs [116,117]. In these composite structures excitonic transfer from the QDs to CNTs could be facilitated, and this was generally studied through steady-state photoluminescence quenching of the QDs when the QDs are in close proximity to the CNTs $[118,119]$. Systematic studies on the separation distance vs. PL quenching of QDs revealed that efficient exciton energy transfer from QDs to CNTs is possible [120]. This exciton transfer increases the photoconductance of the CNTs, which could be beneficial for light-harvesting or lightdetection systems [121]. Recently, the NRET process was accomplished from QDs into several carbon-based nanostructures, including graphene oxide [122], graphite [122], carbon nanofiber [122] and even amorphous carbon thin films [123].

\subsubsection{QD-organics excitonic interactions}

Colloidal QDs are solution-processable materials, which make them compatible with the majority of the organic materials, such as conjugated polymers, dyes and proteins. These QD-organic hybrid nanocomposites find applications in bioimaging and sensing, light-emitting devices (LEDs and lasers) and photovoltaics [53, 54, 124, 125]. In addition, such inorganic-organic composites offer rapid and inexpensive processing techniques (roll-to-roll processing), even on flexible substrates. In this part of the review, we will focus on the excitonically tailored QD-organic composite material systems. Organic materials have active excitonic properties due to the strongly bound nature of the excitons, which are called Frenkel excitons. There is a recent review paper on the excitonic interactions among organic systems [126].

Integrating QDs into conjugated polymers is a common technique for preparing solid-state films of the QDs. The excitonic interactions make these nanocomposites interesting for light generation due to the possibilities of combining the better mechanical and electrical properties of the conjugated polymers with the better optical properties of the QDs. First, Colvin et al. demonstrated a conjugated polymer-QD-based LED [52], which utilized a conjugated polymer as a host charge-transporting matrix. Later, exciton transfer from the conjugated polymers to QDs was identified as a possible scheme for the excitation of the QDs for light-emitting devices [127]. Spectroscopic evidence of this type of exciton transfer has been reported by several groups. Anni et al. demonstrated that the blue- emitting polyfluorene-type conjugated polymer can transfer the optically created excitons into the visible emitting $\mathrm{CdSe} / \mathrm{ZnS}$ core/shell QDs via FRET [128]. Similarly, exciton transfer was reported for infrared-emitting PbS QDs composed of different conjugated polymers [129-131]. Following these initial reports, several studies have focused on developing a deeper understanding of the excitonic processes between conjugated polymers and QDs [132-134]. Stöferle et al. demonstrated that diffusion of the exciton in the conjugated polymer is a vital process for NRET to occur from conjugated polymers to QDs, especially at low QD loading levels in the polymeric films [135]. Lutich et al. revealed the excitonic interactions in an electrostatically bound QD-conjugated polymer hybrid in solution phase; although there is a type II band alignment in the QD-conjugated polymer composite, the dominant excitonic process is found to be NRET rather than charge transfer or Dexter energy-transfer processes [136]. Figure 5 presents the time-resolved fluorescence decay of the donor polyelectrolyte poly[9,9-bis(3'-((N,N-dimethyl)-N-ethylammonium)-propyl)-2,7-fluorene-alt-1,4-phenylene] dibromide (PDFD) polymer and acceptor CdTe QDs that have negatively charged ligands before and after the integration in solution phase. The PDFD conjugated polymer has a single exponential lifetime in the absence of the acceptors, but a double-exponential fit could only account for the measured decay curve in the presence of the acceptors. The newly appeared decay path has the same lifetime scale as the exciton feeding process in QDs (see Fig. 5 top), which confirms that the excitons are transferred from the PDFD to the QDs. The efficiency of the NRET process was measured to be $70 \%$. Ultimately, the interaction zone of the long-range NRET and short-range Dexter energy transfer can be seen in Fig. 5 (bottom).

The exciton transfer dynamics were also modified due to the architecture of the inorganic-organic nanostructure, where a LBL-deposited hybrid assembly of CdTe-QDs and polyelectrolyte conjugated polymer, showed suppression of nonradiative channels in the polymer [137]. Furthermore, in the conjugated polymer-QD mixtures, one important effect should be considered; the phase segregation of the constituent materials. This segregation is observed in the mechanically blended QD-conjugated polymer systems such that the QDs tend to form aggregates in the solidstate films. The phase segregation restricts the NRET in the QD-polymer films via suppressing the interaction volume. Therefore, it is crucial to control the nanoscale interactions in these hybrids to achieve the desired excitonic operation [138-140].

Small organic molecules are frequently employed in the OLED and OPV architectures as electron/hole transport or emissive layers. Furthermore, these molecules are employed in the QD-based LEDs; therefore, it is important to understand the excitonic interactions between these small organic molecules and the QDs to engineer QDbased LEDs [53, 141, 142]. The charge injection from the adjacent organic layers into the QDs is not efficient due to unbalanced injection, leading to Auger recombination in the QDs [143]. By contrast, excitonic injection 

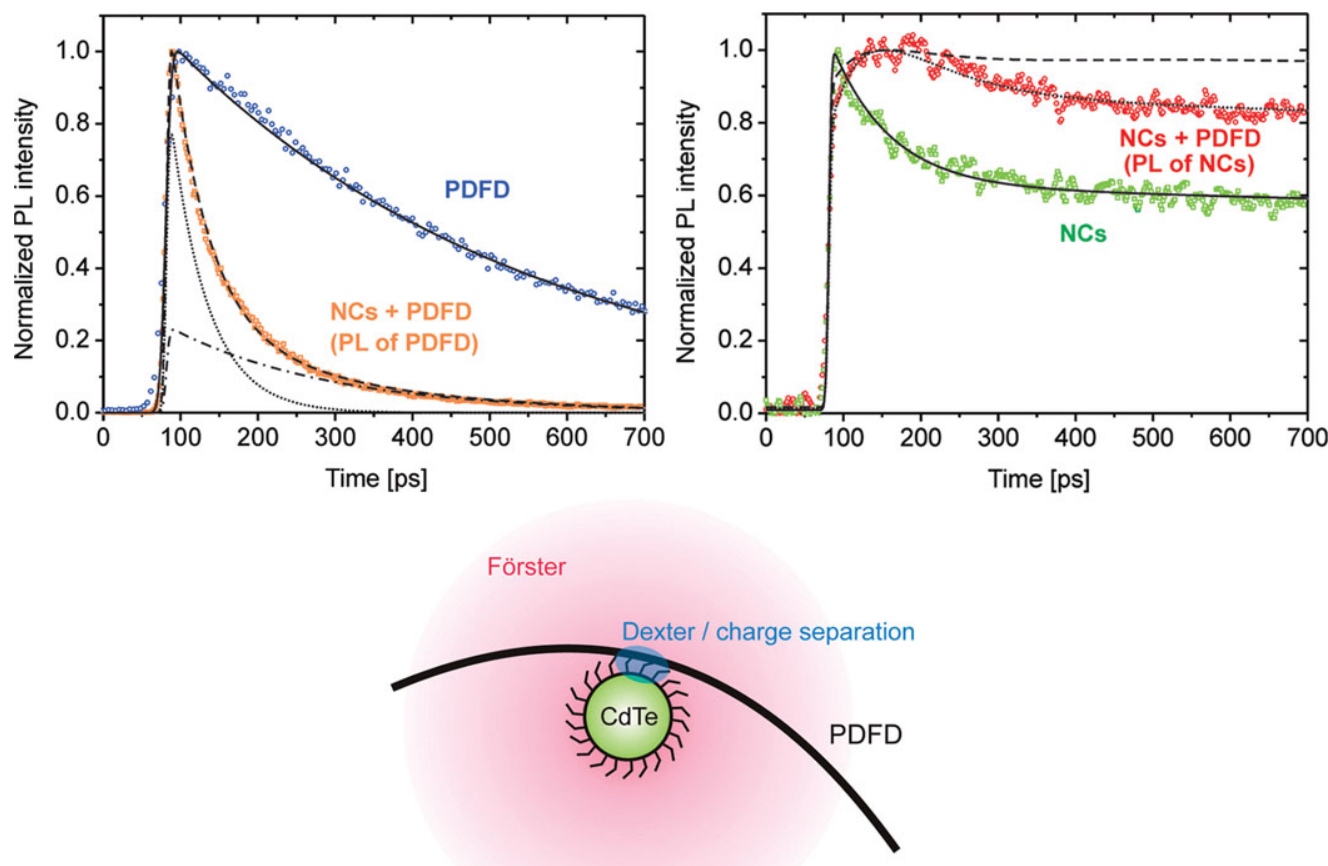

Figure 5 Time-resolved fluorescence decays for the donor PDFD and acceptor CdTe QDs in the PDFD-CdTe QD hybrid nanocomposite (solution phase) are shown before and after the incorporation. The decay of the PDFD becomes significantly faster upon QD integration due to the efficient NRET. The decay of the QD shows the exciton feeding on the same time scale of the NRET via slowing in the decay curve. Although there is a type-II band alignment in the nanocomposite, the dominant excitonic interaction is NRET with $70 \%$ efficiency. Other excitonic interactions, such as Dexter energy transfer and charge separation, are limited due to their short-range operation, as shown in the bottom schematic of the hybrid. [Reprinted (adapted) with permission from ref. [136] (Copyright 2009 American Chemical Society).]

could resolve this charging and subsequent Auger recombination problem. Therefore, maximizing the excitonic injection from the adjacent small organic molecule layers into QDs is vital. For example, TPBi (1,3,5-tris(Nphenylbenzimidizol-2-yl)benzene), which is one of the most frequently used electron-transport and hole-blocking layer, was shown to possess an exciton transfer efficiency up to $50 \%$ into core/multishell CdSe/CdS/ZnS QDs [144]. The engineering of the shell composition and thickness to match with the TPBi emission was shown to lead to strengthened excitonic interactions. Later, TPD ( $\mathrm{N}^{\prime}$-diphenyl-N, $\mathrm{N}^{\prime}$-bis(3-methylphenyl) 1, 1'-biphenyl-4, 4' diamine) and TcTa $\left(4,4^{\prime}, 4^{\prime \prime}\right.$-Tri(9-carbazoyl)triphenylamine), which are widely used for hole-transport purposes, were also shown to have a large exciton transfer capability when they are adjacent to QDs [145]. Furthermore, phosphorescent molecules, where heavy-metal atoms create a strong spin-orbit coupling and intersystem crossing, have highly emissive triplet states. These phosphorescent molecules are promising candidates for exciton injection to QDs. It was demonstrated that an iridium complex phosphorescent molecule called $\operatorname{Ir}(\text { ppy })_{3}$ (fac-tris(2-phenylpyridine)iridium) can enhance the steady-state $\mathrm{PL}$ emission of the $\mathrm{CdSe} / \mathrm{ZnS}$ core/shell QDs in a bilayer film structure of QDs and $\operatorname{Ir}(\mathrm{ppy})_{3}$ in CBP (4,4'-N,N'-dicarbazolyl-1,1'-biphenyl) [146]. However, the underlying physics of the exciton transfer between the QDs and organic molecules is still unknown, whether the main transfer route is through NRET or Dexter transfer. However, this scheme was applied to hybrid QD-LEDs and descent enhancements were observed in the external quantum efficiencies (EQEs) of the devices [147-149]. Although there are concomitant enhancements in the device performances, the efficiencies are still well below the EQEs of those of only phosphorescent devices ( $>20 \% \mathrm{EQE}$ ). More suitable architectures, rather than simple bilayers of the QDs and phosphorescent molecules, are desired for efficient excitonic operation.

Additionally, bioconjugates of the QDs with proteins have been investigated for imaging, labeling and sensing applications in biology [124]. These bioconjugates of the QDs are excitonically active such that the QDs can function as both the exciton donor and acceptor [150-155]. These hybrids could be promising for future lighting and lightharvesting systems. For example, chemical and biological systems can produce light upon molecular-level interactions so-called chemiluminescence and bioluminescence. These systems can be used as novel light-generation structures with the incorporation of QDs due to their superior color control and tuning abilities. Through bio- or chemiluminescence resonance energy transfer (BRET or CRET), 


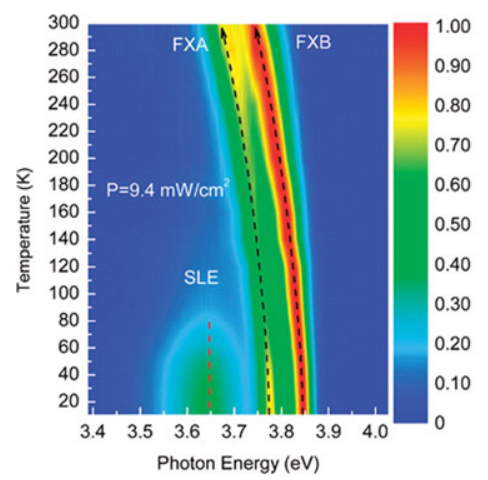

the excitation energy created in the bio- or chemiluminescent system can be transferred to the QDs [156-158]. These initial demonstrations were targeted for applications as external light sources for bioimaging and sensing applications. Furthermore, electrically activated chemiluminescence and the transfer of the excitons to QDs were shown to be favorable for sensing applications $[159,160]$. In addition to bioconjugates, QD-dye hybrids show promise for biological sensing and labeling applications. NRET between luminescent dyes and QDs have been studied in detail to elucidate the effects of concentration, shape and structure of the hybrids [161-165].

\subsection{Excitonic interactions in Qwires}

The excitonic operation is also prevalent in semiconductor Qwires, and many groups have studied the excitonic properties of various Qwire systems in the pursuit of obtaining a better understanding of the photonic properties of the Qwires. Excitons in the Qwires are confined in two dimensions (2D), and the properties of these excitons are generally less pronounced in the optical properties of the Qwires as compared to those observed in QDs. This is because it is not easy to fabricate Qwires with diameters smaller than $10 \mathrm{~nm}$ with the available physical and chemical vapor deposition techniques whereas colloidally synthesized QDs can be made quite small - on the order of a few nanometers in diameters; therefore, QDs exhibit much stronger quantum confinement than the current Qwires [166-168]. To attain the large binding energies required for creating stable excitons, the physical dimensions of the Qwires should be made smaller than the bulk exciton Bohr radius.

To date, Qwires of a broad range of semiconductor materials have been synthesized and their excitonic features have been confirmed and investigated using optical spectroscopy. For Qwires, which have poor quantum confinement and small bulk exciton binding energy, excitons are not stable and they are dissociated into free carriers at room temperature (i.e. $k_{\mathrm{B}} T \sim 25 \mathrm{meV}>E_{\mathrm{B}}$ ). In this case, the Qwires need to be cooled to observe the excitonic features in their optical properties. However, materials with large bulk exciton binding energies such as $\mathrm{ZnO}, \mathrm{ZnS}$ and $\mathrm{CdS}$ exhibit room-temperature excitonic behavior with little help from the quantum-confinement effects. Furthermore, for Qwires of, for example, CdSe, InP and GaAs exciton binding energies were made nearly an order of magnitude larger than the bulk binding energies due to the quite small radii $(<5 \mathrm{~nm})$ of the Qwires $[167,168]$.

The crystal quality and defects are important for the optical and excitonic properties of the Qwires. Nonradiative relaxation channels due to the presence of defects should be suppressed. Therefore, it is crucial to have high crystal quality Qwires with low defect densities [169]. In addition, the temperature-dependent optical properties of $\mathrm{ZnS}$ Qwires were investigated by Chen et al. Figure 6 presents the excitonic emission spectrum at $10 \mathrm{~K}$ with the mapping of the excitonic emission peaks as a function of temperature. Excitonic operation, which is crucial to maintain the efficient radiative recombination of the optically pumped $\mathrm{ZnS}$ Qwires, was shown to be apparent at room temperature due to the large exciton binding energy of the $\mathrm{ZnS}$ Qwires. These ZnS Qwires are promising new materials for UV-emitting LEDs and lasers [170].

One promising use of the excitonic phenomena in Qwires is in stimulated emission generation and lasing. Since excitons are bound entities and tend to radiatively recombine, they lead to strong light emission properties in the materials. In combination of this excitonic nature with the unique structural advantages of the Qwires such as light confinement, Qwires systems were shown to be suitable for lasing applications. Huang et al. first showed the applicability of $\mathrm{ZnO}$ Qwires as an active gain medium for optically pumped random nanolasers [171]. Lasing was exhibited at room temperature from the fabricated $\mathrm{ZnO}$ Qwires, which exhibit strong exciton and photon confinement properties. In 2005, Agarwal et al. demonstrated strong lasing emission from high-quality single-crystal CdS Qwires [172]. $\mathrm{CdSe}$ Qwires can also be utilized as room-temperature lasing media at near-IR wavelengths. Although the excitonic operation was strong at room temperature in these Qwires, weak phonon-exciton interactions were demonstrated to assist the room temperature lasing [173]. Xu et al. revealed an amplified spontaneous emission (ASE) buildup in the network of one-dimensional CdS-based nanobelts. The defects in the nanobelts were revealed to alter the excitonic processes by creating bound excitons. Engineering these defects was shown to provide fine control of the ASE threshold due to the interplay between the bound exciton density and Auger-recombination kinetics [174]. Qwires, which have 

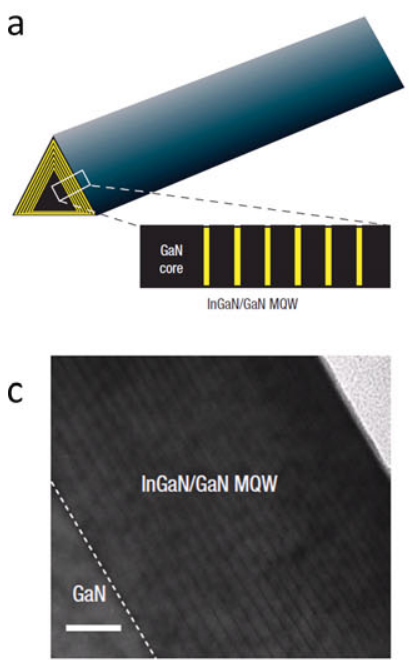

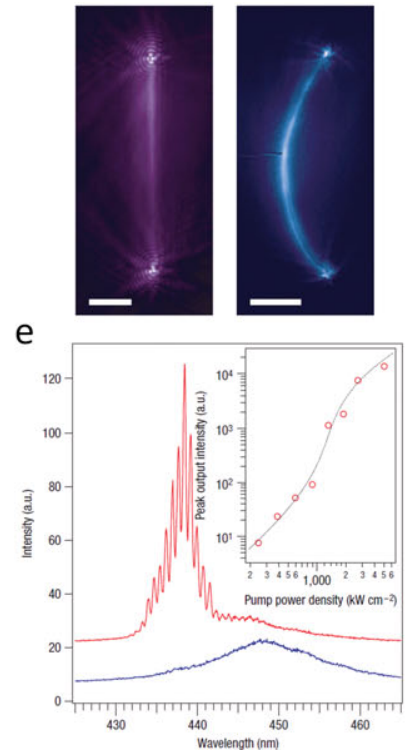
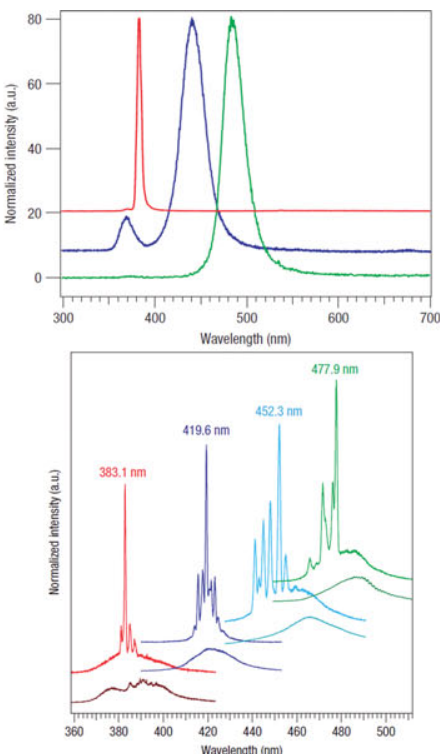

Figure 7 (a) Schematic diagram of GaN/AIGaN multicore/shell Qwires. The core is composed of GaN Qwires. The shell is composed of multiple GaN/AIGaN QW. (b) Dark-field cross-sectional STEM images of multicore/shell Qwires with 3 Qwell layers. The scale bars are $20 \mathrm{~nm}$. (c) Bright-field TEM image of a multicore/shell Qwires with 26 Qwell layers. (d) Zoomed in TEM image of the quantum wells. The scale bar is $10 \mathrm{~nm}$. (e) Emission and lasing properties of as-grown multicore/shell Qwires. The PL image and PL emission band varies as the component of the shell (left-right, upper). With GaN/AIGaN Qwells shell as gain media and GaN core as cavity, the multicore/shell structures can serve as a microlaser (left, bottom). The lasing wavelength is effectively tuned from 365 to $484 \mathrm{~nm}$ (right, bottom). Reprinted by permission from Macmillan Publishers Ltd: Nature Materials (ref. [176]), copyright (2008).

a naturally formed flat facet, are also good candidates for optical amplifications as semiconductor gain media [57]. In addition to laser diodes, Qwires have also been employed in LEDs, photodetectors and FETs [60].

Structured Qwires have also been introduced towards obtaining high-quality and functional 1D systems. In these Qwires, alternating materials (i.e. at least two or more) are grown either on the axial direction (end-to-end stacking) or on the radial direction (core/shell-like stacking) [175]. These heterostructured Qwires were shown to generate $\mathrm{p}-\mathrm{n}$ junctions on single wires. Furthermore, core/shell Qwire heterostructures have shown advantageous properties for excitonic control because the cores can be highly confined and passivated via shell growth such that 1D excitonic operation can be efficiently preserved. Recently, Qian et al. demonstrated GaN/AlGaN multicore/shell Qwire heterostructures, in which the $\mathrm{GaN}$ core is surrounded with highly uniform GaN/AlGaN multiple QWs shell (see Fig. 7a) $[45,176,177]$. The TEM studies revealed that the growth of multiple QWs based on a GaN core is epitaxial and dislocation-free. Furthermore, the emission and lasing wavelength of the multicore/shell Qwires, which is determined by the $\mathrm{AlGaN}$ component, can be tailored over a wide range at room temperature (365-494 nm), as shown in Fig. 7b. In addition, the photon confinement and consequently the mode volume in the $\mathrm{GaN}$ core can be tuned by the numbers and structures of the QWs shell. This heterostructure could be suitable as a lasing medium due to the exciton and photon confinement effects.
Zinc oxide $(\mathrm{ZnO})$ is an emerging semiconductor material with a very high bulk exciton binding energy $(\sim 60 \mathrm{meV})$. Therefore, the excitonic features can be easily observed in the optical properties, even at room temperature. This property led to the development of $\mathrm{ZnO}$-based optoelectronic devices in the last decade. $\mathrm{ZnO}$ Qwires were employed in LEDs by combining different materials such as p-Si and p-GaN with n-ZnO Qwires [178-183]. Zimmler et al. demonstrated a single $\mathrm{ZnO}$ Qwire LED, where EL spectrum was investigated as a function of temperature, and at low temperatures (7-200 K), the emission spectra of the LED was dominated by strong excitonic emission [184]. Recently, using the piezoelectric characteristics of $\mathrm{ZnO}$, mechanical deformation was shown to modify the excitonic features in the emission spectra $[185,186]$.

\subsubsection{Excitonic interactions in carbon nanotubes}

Carbon nanotubes (CNTs) make an interesting class of 1D materials that exhibit unique Qwire properties. A recent review on the single-walled CNTs (SWCNTs) summarizes the excitonic properties in the CNTs [187]. Experimental and theoretical studies have shown that the optical properties of CNTs are governed by strong excitonic behavior [188-190]. For example, the excitons in semiconducting single-walled CNTs (SWCNTs) were observed to be strongly bound due to the large exciton binding energies $(\sim 300-600 \mathrm{meV})$ [191], and these excitonic features are 

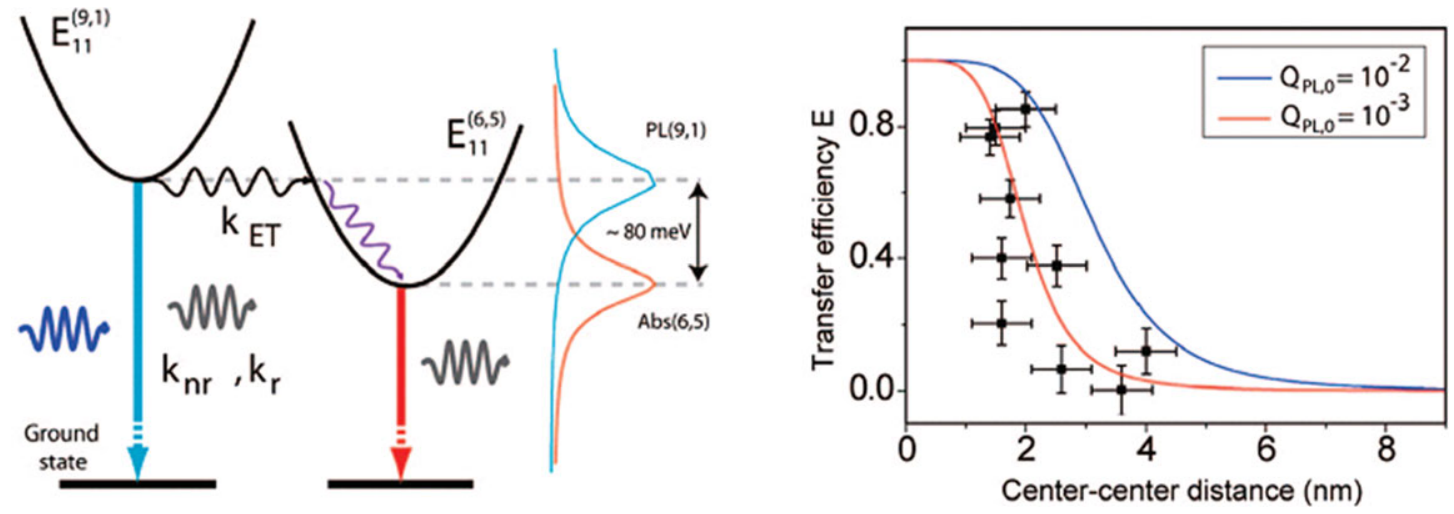

Figure 8 Exciton transfer via NRET in the SWCNT bundles. Similar to NRET in QD assemblies, NRET can occur in the CNT bundle from a larger-bandgap CNT to a smaller-bandgap CNT. Energy-transfer efficiency was plotted as a function of separation distance between the two interacting CNT for the two cases of PL QY. For the separation distance NRET becomes efficient. [Reprinted (adapted) with permission from ref. [200] (Copyright 2008 American Chemical Society).]

dominant in the optical absorption of the CNTs [190]. This active excitonic operation in SWCNTs makes them auspicious materials for light-harvesting applications. Consequently, semiconducting SWCNTs were employed as nearIR light harvesters by hybridizing them with $\mathrm{C}_{60}$ molecules in a bilayer architecture. Exciton dissociation was demonstrated at the proposed $\mathrm{CNT} / \mathrm{C}_{60}$ interface, although $\mathrm{CNT}$ excitons have quite large binding energies [192]. To assess the device's performance, carefully sorted semiconducting SWCNTs were used as the active absorber layer with a film thickness less than the exciton diffusion length of the CNTs such that excitons can easily become dissociated [192]. In addition, exciton diffusion and mobility of the excitons were measured in the SWCNTs via photoluminescence quenching experiments, and the results revealed that the exciton diffusion lengths are up to $250 \mathrm{~nm}$, which is along the nanotube axis [193]. With the goal of better light-harvesting systems based on CNTs, Wang et al. investigated multiexciton generation in the SWCNTs via high-energy photon irradiation (i.e. 355 and $400 \mathrm{~nm}$ ). The absorption of the high-energy photons was shown to create multiexcitons in the semiconducting SWCNTs with a carrier-multiplication threshold that is close to the theoretical limit ( $h v \sim 2 E_{\mathrm{g}}$ ) [194]. Furthermore, Auger recombination was shown to be highly effective in these $1 \mathrm{D}$ confined CNTs due to the strongly bound nature of the excitons, similar to the case in the QDs [194]. This recombination could limit the effectiveness of light-harvesting systems due to the loss of excitons through thermalization.

Furthermore, SWCNTs were observed to emit light via radiative recombination of the excitons [195]. However, not all the excitons can decay radiatively because of the existence of the so-called dark excitons [189]. The infrared emission from the SWCNTs was later employed for electroluminescent devices [196-198]. EQEs of these proposed devices were measured to be on the order of $10^{-4}$. This poor performance was attributed to the poor PL QY of the CNTs, which is on the order of 0.01 [196]. Recently, the use of asymmetric contact was proposed to enhance the CNT- based LEDs, which were shown to exhibit narrow excitonic emission at $0.9 \mathrm{eV}[197,198]$.

In addition to these excitonic features in the optical properties of the CNTs, the inter-CNT excitonic interactions (exciton transfer) have also been investigated in the literature. Exciton energy transfer in the form of longrange NRET was shown in the bundles of the SWCNTs [199-202]. Figure 8 shows the mechanism for NRET in the SWCNT bundle. SWCNT with a larger bandgap can transfer the exciton to another SWCNT that has a smaller bandgap. The NRET efficiency was plotted as a function of the separation distance between the donor-acceptor CNTs (see Figure 8). For distances equal to or less than $3 \mathrm{~nm}$, efficient NRET could be observed. The primary limitation behind the observed small Förster radius (2-3 nm) was attributed to the very low photoluminescence of the CNTs, which makes them inefficient exciton donors. In addition to SWCNTs, exciton transfer was observed in the doublewalled CNTs (DWCNTs) assemblies, where energy transfer occurs in both intra- and inter-CNTs [203,204].

\section{Excitonic interactions beyond the Förster limit}

\subsection{Plasmon-exciton interactions for enhanced excitonic coupling (plexcitons)}

Plasmonics is an emerging field in nanophotonics and has applications ranging from solar cells to photonic circuits. Plasmons are the collective oscillations of electrons in the metals. Investigation of the plasmon-exciton couplings (called plexcitonics) has created an interest in the scientific community. Several groups have studied fluorescent materials attached to metallic nanoparticles in efforts to elucidate this complex interaction [205-209]. It was shown that nearby plasmonic oscillations could modify the radiative recombination of the fluorescent material. 
Furthermore, emission of the emitter could also be enhanced due to the competition of the radiative rate over the nonradiative ones. Furthermore, when the separation is sufficiently small (sub-5 nm), nonradiative energy transfer becomes dominant. This nonradiative energy transfer leads to the strongest energy transfer from the fluorophores to plasmons; consequently, the fluorophore emission is severely quenched. A considerable number of experimental works have reported that the intensity of emission in this sub-5 $\mathrm{nm}$ region monotonically varies with decreasing separation [210-213]. Recently, Peng et al. [214] found that the spectral overlap between the plasmon and emission bands also plays an important role in the above energy-transfer process, which can lead to a minimal emission intensity of $\sim 2 \mathrm{~nm}$.

In considering the effect of plasmon enhancement of emission from semiconductor elements of a nanocrystal superstructure, it is important to look at the exciton-plasmon and photon-plasmon resonance conditions, which can be formulated in the following, simple way [205]:

$$
\omega_{\text {exciton }} \approx \omega_{\text {plasmon }} \text { and } \omega_{\text {photon }} \approx \omega_{\text {plasmon }},
$$

where the frequencies involved are related to excitons in a semiconductor component, plasmons in metallic components, and photons of incident light. Under illumination of a given intensity, an exciton-plasmon nanocrystal complex constructed by using the above conditions (4) can exhibit strongly enhanced emission. Under the excitonplasmon resonance $\left(\omega_{\text {exciton }} \approx \omega_{\text {plasmon }}\right)$, the enhancement comes from an increased probability for an exciton to emit a photon since an exciton becomes coupled with a plasmon and, in this way, acquires an enhanced optical dipole. Under the second condition ( $\left.\omega_{\text {photon }} \approx \omega_{\text {plasmon }}\right)$, excitons inside a hybrid semiconductor-metal system acquire an increased absorption cross section that also leads to amplified emission. Interestingly, semiconductor emitters and metal plasmonic amplifiers can be made of various shapes and dimensionalities. This is thanks to a wide variety of possibilities enabled by current technology, for example, including nanocrystal bioassembly in a liquid phase. Two examples of such nanowire-nanocrystal structures (CdTe$\mathrm{Au}$ and $\mathrm{CdTe}-\mathrm{Ag}$ ) with strongly enhanced PL emissions were reported and described in Refs. [215,216]. Different metals can sustain different surface plasmon resonances, at $\sim 500$ and $\sim 400 \mathrm{~nm}$ for $\mathrm{Au}$ and Ag nanocrystals, respectively. These plasmon resonances were employed for a realization of the two resonance conditions (4) using CdTe Qwires as emitters. Consequently, the structures designed according to the conditions (4) worked well as plasmonic amplifiers for the exciton emission from the semiconductor Qwires. Figure 9 shows one realization of a structure with the photon-plasmon resonance, which involves $\mathrm{CdTe}$ nanocrystals and $\mathrm{Ag}$ nanoparticles [216]. As the $\mathrm{CdTe}-\mathrm{Ag}$ hybrid system is formed in solution via biolinkers, the photoluminescence excitation spectra (PLE) at the CdTe peak emission wavelength exhibits strong enhancement for the spectral region around the plasmon resonance of the $\mathrm{Ag}$ nanoparticles, as shown in Fig. 9.

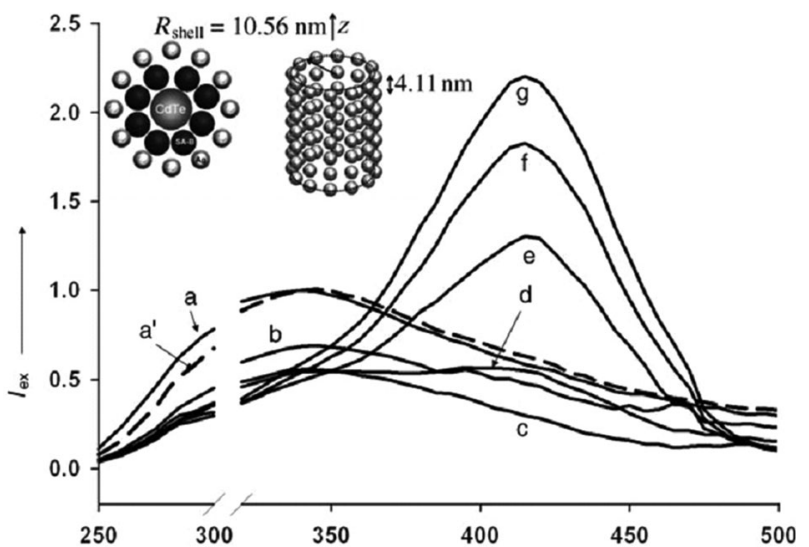

Figure 9 Schematics of the CdTe-Ag nanowire-nanocrystal structure with enhanced emission properties due to the photonplasmon resonance. The structure was assembled in a solution using special biolinkers (SA-B). The plot shows the photoluminescence excitation (PLE) spectra at the peak emission wavelength of the CdTe nanocrystals. After every $10 \mathrm{~min}$, PLE spectrum is measured for $(\mathrm{a}) \rightarrow(\mathrm{g})$. As the CdTe-Ag hybrid is formed in solution, the significant enhancement in PLE signal is observed at $\sim 420 \mathrm{~nm}$. The schematic shows a cross section with a central CdTe NW and an Ag-nanoparticle shell. Reprinted by permission from Wiley (ref. [216]), Copyright $\odot 2006$ Wiley-VCH Verlag $\mathrm{GmbH} \& \mathrm{Co}$. KGaA, Weinheim

NRET between quantum-confined structures could also be modified in the presence of plasmonic coupling. The phenomenon of the NRET enhancement via plasmonic coupling was theoretically described for the case of QDs by Govorov et al. [217] Later, it was experimentally demonstrated that localized plasmons could enhance the dipoledipole coupling. In turn, NRET could be enhanced, even at larger separation distances [218,219]. Figure 10 presents the steady-state and time-resolved signatures of the NRET enhancement via localized plasmon oscillations of $\mathrm{Au}$ nanoparticles. This enhancement was corroborated via both spectroscopic measurements and detailed modeling. The Förster radius was observed to almost double due to the antenna effect of the Au nanoparticles for the case of NRET from green-emitting to red-emitting CdTe QDs in a LbL assembled architecture. Furthermore, using a plasmonic cavity coupled to QDs polarized emission was detected from the isotropic QD emitter, which is promising for polarizedlight generation [220-222].

\section{Conclusions}

In summary, we reviewed the excitonics of quantumconfined dots and wires for their optical properties important for lighting and displays. First, we discussed the basic excitonic processes, which are frequently observed in the assemblies of the QDs and Qwires such as nonradiative energy transfer, Dexter energy transfer and exciton diffusion. Then, excitonic interactions were reviewed in the mixed hybrid systems of the QDs. Subsequently, current 

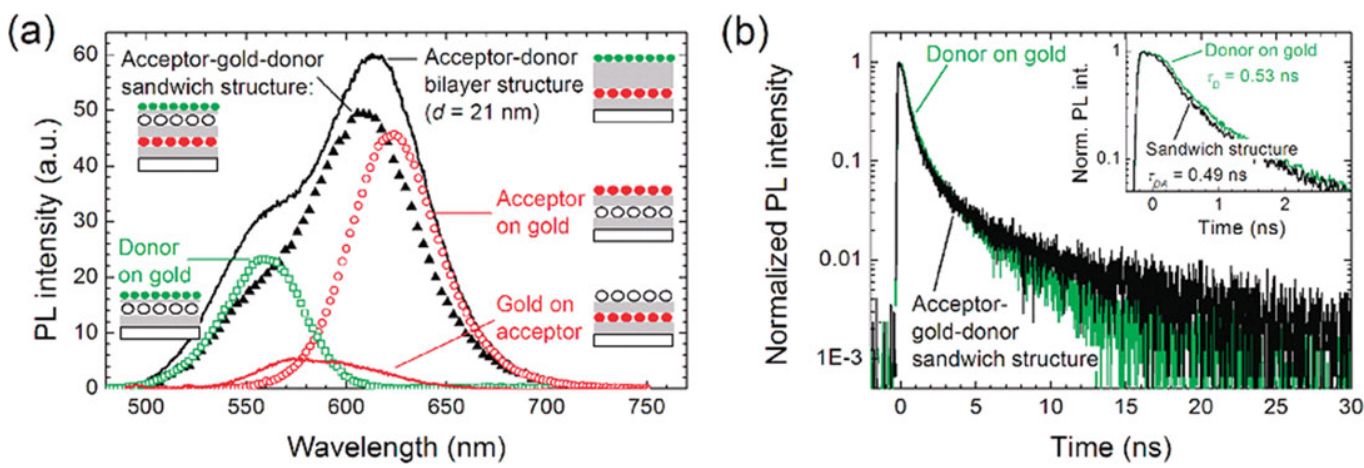

Figure 10 Enhancement of the NRET in the CdTe QD-Au nanoparticle layer-by-layer coated system investigated using (a) steadystate and (b) time-resolved spectroscopy. (a) Using four different negative control samples (donor on gold, acceptor on gold, gold on acceptor and acceptor-donor bilayer structure) and the working sample (acceptor-gold-donor sandwich structure) steady-state emission properties of the QDs were compared. (b) In the time-resolved decay curves, modification of the exciton lifetime of the donor QDs at a large separation distance was shown in the presence and absence of the gold nanoparticles. The acceptor-gold-donor structure exhibits faster donor lifetime as compared to the case of donor on gold, which is attributed to the enhanced NRET due to the gold nanoparticles. [Reprinted (adapted) with permission from ref. [219] (Copyright 2011 American Chemical Society).]

understanding of the excitonics in Qwires and promising applications such as Qwire lasers and LEDs were reviewed. After that, exciton-plasmon interactions and possible consequences on the enhanced optical properties and energytransfer abilities were outlined. Finally, we highlighted several future perspectives and technological and scientific challenges towards excitonically engineered QD and Qwire systems for lighting and light-harvesting applications.

\section{Future challenges and research opportunities}

Our understanding of the excitonic interactions in quantumconfined structures has evolved considerably in recent years. Being able to engineer the excitonic processes in the structures of the QDs and QWs enabled the performance of the already existing devices to be boosted, such as color conversion LEDs of QDs or lead to novel devices such as Qwire lasers, which are all important for efficient light-generation systems. However, the development of these excitonically controlled devices and systems will require deeper understanding of the nanoscale interactions of these confined systems along with further developments in materials and processing techniques.

\subsection{Excitonic QD LEDs}

Exciton energy transfer facilitated QD-based LEDs started to arise as an important class of LEDs. Similarly, QD-based LEDs, which employ these QDs in the electrically driven active layers are promising for general lighting purposes and displays. However, these QD-LEDs suffer from poor charge injection and subsequent low EQEs due to the organic insulating ligands of the QDs. Therefore, excitonic injection could possibly address the current problems in these charge-driven QD-LEDs via pumping the QDs by NRET in addition to or instead of direct charge pumping. However, the challenge is to find a suitable material that possesses good charge transport, exciton formation and exciton transfer properties together. For example, quantum wells have been suggested as the appropriate exciton donors, since they have good charge transport and exciton formation properties, but it was difficult to intimately integrate the QDs so close to the quantum wells such that exciton transfer was limited in these hybrid systems [91, 104]. Another candidate as the proper donor material would be organic materials as conjugated polymers and phosphorescent molecules, which are frequently used in organic LEDs efficiently. Yet, a fully excitonic QD-LED has not been shown yet. In addition, Qwire-QD excitonic couplings for lighting might have promising results as compared with QW-QD systems. It is a challenge to design a hybrid system of Qwires integrated with QDs for light-emitting purposes, which might have applications in excitonically tunable LEDs and lasers.

\subsection{Excitonic Qwire Lasers}

Excitonic emission in a material is favorable for achieving stimulated emission because excitons have a strong tendency to radiatively recombine. However, ensuring stable excitons at room temperature is a challenge for various material systems due to their small exciton binding energies, which require highly quantum-confined Qwires. That is, on the other hand, still technologically difficult and a challenge to synthesize Qwires that have very small radii.

Acknowledgements. We gratefully thank Professor Charles Lieber of Harvard University very much for his valuable assistance and useful suggestions. This work was supported by the Singapore National Research Foundation under NRF-CRP-62010-02 and NRF-RF-2009-09 and also, in part, by the Singapore Agency for Science, Technology and Research (A*STAR) SERC under Grant No. 112120 2009. H.V.D. acknowledges 
support from ESF-EURYI and TUBA-GEBIP. A.O.G. acknowledges support from DoD within the MURI program and NSF (USA) and Volkswagen Foundation (Germany).

Received: 13 February 2013, Revised: 18 April 2013, Accepted: 22 April 2013

Published online: 25 May 2013

Key words: Excitonics, quantum dots, quantum wires, quantum wells, organics, carbon nanotubes, exciton transfer, nonradiative energy transfer, excitonic interactions, Förster resonance energy transfer, plexcitons, lighting.

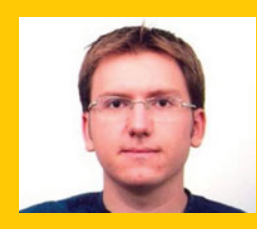

Burak Guzelturk received his M.S. degree in electrical and electronics engineering in 2011 from Bilkent University, Turkey. He is pursuing a Ph.D. degree under the supervision of Prof. Demir in the Devices and Sensors Group at the $\mathrm{Na}$ tional Nanotechnology Research Center (UNAM) at Bilkent University. He is working towards developing excitonically enabled colloidal quantum-dot-based systems for light-generation and light-harvesting applications.

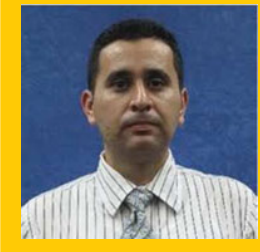

Dr. Pedro Ludwig Hernandez Martinez received his M.S. and Ph.D. degrees in physics from Ohio University, USA, in 2007 and 2010, respectively. He is currently a postdoctoral research fellow working with Prof. Demir at the Luminous! Center of Excellence for Semiconductor Lighting and Displays at NTU Singapore and a visiting postdoctoral researcher at Bilkent University. His research work includes the theoretical understanding and modeling of the excitonic phenomena at the nanoscale.

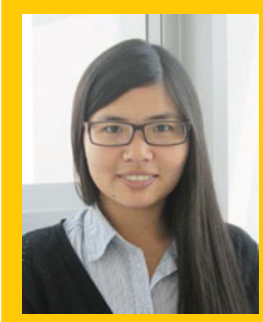

Dr. Qing Zhang received her Ph.D. degree in physics from Tsinghua University in 2011. She is currently a postdoctoral research fellow at NTU Singapore at Nanyang Technological University. The areas of her research focus include optical spectroscopy, optical/excitonic properties of semiconductors, plasmonics in metallic stuctures including exciton-plasmon interaction and micro/ nanolasing.

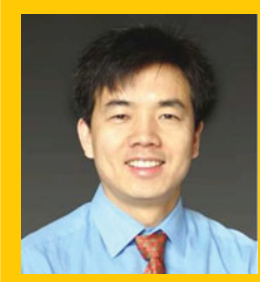

Dr. Qihua Xiong obtained his Ph.D. degree in materials science at the Pennsylvania State University, USA, in 2006. He is currently Nanyang Assistant Professor at Nanyang Technological University and an NRF Fellow of Singapore. His research covers rational synthesis of functional semiconductor nanomaterials, systematic investigations on their physical properties in quantum size regime and practical applications in nanoelectronics, nanophotonics and nanobiotechnology.

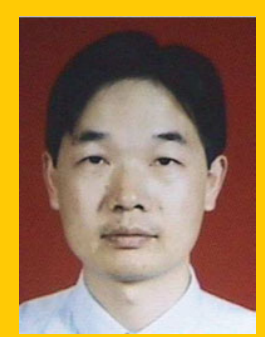

Dr. Handong Sun received his M.S. and Ph.D. degrees from Huazgong University of Science \& Technology, China, and Hong Kong University of Science and Technology, Hong Kong, respectively. $\mathrm{He}$ is currently an associate professor at Nanyang Technological University, Singapore. His research interests include optoelectronic materials and devices, semiconductor physics, optical spectroscopy, and nanomaterials and technology.

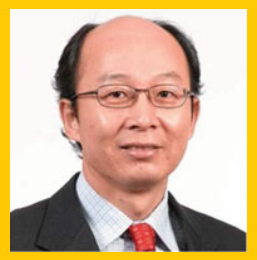

Dr. Xiao Wei Sun obtained his PhD degrees from Tianjin University, China, and Hong Kong University of Science and Technology, Hong Kong, in 1996 and 1998, respectively. He is a professor at Nanyang Technological University, Singapore, and is the Deputy Director of the Luminous! Center of Excellence for Semiconductor Lighting and Displays. His research interests focus on semiconductor physics and devices, display technologies, and nanotechnology.

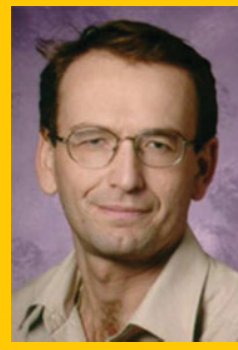

Dr. Alexander O. Govorov received his PhD from the Institute of Semiconductor Physics in Russia in 1991. Currently he is a professor at the department of physics and astronomy at Ohio University, USA. His research interests include theoretical condensed-matter physics including physics of semiconductor nanostructures, optical and transport phenomena, many-body effects, quantum phenomena and nanoscience.

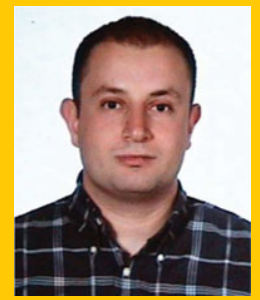

Dr. Hilmi Volkan Demir obtained his M.S. and Ph.D. degrees in electrical engineering at Stanford University in 2000 and 2004, respectively. He is an NRF Fellow of Singapore and Nanyang Associate Professor at NTU Singapore, and serves as the Director of the Luminous! Center of Excellence for Semiconductor Lighting and Displays. Concurrently, he is the EURYI Associate Professor at Bilkent University Turkey. His research work includes the science and technology of excitonics and plasmonics for light generation and harvesting; nanocrystal optoelectronics for semiconductor lighting.

\section{References}

[1] T. Förster, Ann. Phys. 437, 55-75 (1948).

[2] T. Förster, Naturwissenschaften 6, 166-175 (1946).

[3] T. Förster, Z. Elektrochem. 53, 93-100 (1949).

[4] T. W. J. Gadella, Chapter 1: Förster resonance energy transfer-FRET what is it, why do it, and how it's done, by R. M. Clegg, Laboratory Techniques in Biochemistry and Molecular Biology, Vol. 33, (Academic Press, Burlington, 2009). 
[5] J. R. Lakowicz, Principles of Fluorescence Spectroscopy, 3rd edn, (Springer, New York, 2010).

[6] L. Stryer and R. P. Haugland, Proc. Natl. Acad. Sci. USA 58, 719-726 (1967

[7] V. M. Agranovich, Y. N. Gartstein, and M. Litinskaya, Chem. Rev. 111, 5179-5214 (2011).

[8] P. L. Hernandez-Martinez, A. Govorov, and H. V. Demir, arXiv:1302.1664 [cond-mat.mes-hall] (2013).

[9] D. L. Dexter, J. Chem. Phys. 21, 836 (1953).

[10] B. Valeur, Molecular fluorescence: principles and applications, (Wiley-VCH Verlag GmbH, Weinheim, 2002).

[11] A. Köhler and H. Bassler, Mater. Sci. Eng. R 66, 71-109 (2009).

[12] J.-L. Bredas and R. Silbey, Science, 323, 348-349 (2009).

[13] L. Novotny and B. Hecht, Princples of Nano-optics, (Cambridge University Press, New York, 2006).

[14] B. A. Gregg, J. Phys. Chem. B 107, 4688-4698 (2003).

[15] N. S. Saricifcti, L. Smilowitz, A. J. Heeger, and F. Wudl, Science 258, 1474-1476 (1992).

[16] B. O'Regan and M. Gratzel, Nature 353, 737-740 (1991).

[17] A. J. Nozik, Chem. Phys. Lett. 457, 3-11 (2008).

[18] M. C. Beard, J. Phys. Chem. Lett. 2, 1282-1288 (2011).

[19] V. I. Klimov, A. A. Mikhailovsky, S. Xu, A. Malko, J. A. Hollignsworth, C. A. Leatherdale, H.-J. Eisler, and M. G. Bawendi, Science 290, 314-317 (2000).

[20] P. Harrison, Quantum wells, wires and dots: theoretical and computational physics of semiconductor nanostructures, (Wiley-Interscience, London, 2005).

[21] B. K. Meyer, H. Alves, D. M. Hoffman, W. Kriegseis, D. Forster, F. Bertram, J. Christen, A. Hoffmann, M. Strassburg, M. Dworzak, U. Haboeck, and A. V. Rodina, Phys. Status Solidi B 241, 231-260 (2004).

[22] S. Hong, T. Joo, W. I. Park, Y. H. Jun, and G.-C. Yi, Appl. Phys. Lett. 83, 4157-4159 (2003).

[23] N. Uzar and M. Ç. Arıkan, Bull. Mater. Sci. 34, 287-292 (2011).

[24] Z. Deng, L. Tong, M. Flores, S. Lin, J.-X. Cheng, H. Yao, and Y. Liu, J. Am. Chem. Soc. 133, 5389-5396 (2011).

[25] R. Tomassi, M. Lepore, M. C. Netti, I. M. Catalano, and I. Suemune, Phys. Rev. B 49, 14367-14371 (1994).

[26] T. Miyajima, F. P. Logue, J. F. Donegan, J. Hegarty, H. Okuyama, A. Ishibashi, and Y. Mori, Appl. Phys. Lett. 66, 180-182 (1995).

[27] P. Lao, Y. Guo, W. Zheng, G. G. Siu, J. Li, and S. Yuan, Appl. Phys. Lett. 65, 2090-2092 (1994).

[28] A. Dong, Colloidal semiconductor nanowires: synthesis, quantum-confinement-effect studies, and heterostructure fabrication, PhD Thesis, Washington University (2007).

[29] J. Singh and S. Kugler, Phys. Status Solidi A 206, 993-996 (2009).

[30] L. V. Titova, T. B. Hoang, H. E. Jackson, L. M. Smith, J. M. Yarrison-Rice, J. L. Lensch, and L. J. Lauhon, Appl. Phys. Lett. 89, 053119 (2006).

[31] J. G. Vilhena, S. Botti, and M. A. L. Marques, Appl. Phys. Lett. 96, 123106 (2010).

[32] A. I. Ekimov, F. Hache, M. C. Schanne-Klein, D. Ricard, C. Flytzannis, I. A. Kudryavtsev, T. V. Yazeva, and A. V. Rodina, J. Opt. Soc. Am. B 10, 100-107 (1993).
[33] N. Pelenakos, Q. Fu, J. Ding, W. Walecki, A. V. Nurmikko, S. M. Durbin, J. Han, M. Kobayashi, and R. L. Gunshor, Phys. Rev. B 41, 9966-9970 (1990).

[34] J. Duan, L. Song, and J. Zhan, Nano Res. 2, 61-68 (2009).

[35] J. Sun, L.-W. Wang, and W. E. Buhro, J. Am. Chem. Soc. 130, 7997-8005 (2008).

[36] G. Steude, B. K. Meyer, A. Göldner, A. Hoffmann, F. Bertram, J. Christen, H. Amano, and I. Akasaki, Appl. Phys. Lett. 74, 2456-2458 (1999).

[37] J. Christen, D. Bimberg, A. Steckenborn, and G. Weimann, Appl. Phys. Lett. 44, 84-86 (1984).

[38] B. Bansal, A. Kadir, A. Bhattacharya, and V. V. Moshchalkov, Appl. Phys. Lett. 93, 021113 (2008).

[39] S. B. Nam, D. C. Reynolds, C. W. Litton, T. C. Collins, P. J. Dean, and R. C. Clarke, Phys. Rev. B 13, 1643-1648 (1976).

[40] B. Bansal, S. Godefroo, M. Hayne, G. Medeiros-Ribeiro, and V. V. Moshchalkov, Phys. Rev. B 80, 205317 (2009).

[41] V. I. Klimov, Semiconductor and Metal Nanocrystals: Synthesis and Electronic and Optical Properties, (Marcel Dekker, NY, USA, 2004).

[42] K. H. Aharonyan and E. M. Kazaryan, Physica E 44, 19241930 (2012).

[43] W. Ma, J. M. Luther, H. Zheng, Y. Wu, and A. P. Alivisatos, Nano Lett. 9, 1699-1703 (2009).

[44] Q. Sun, Y. A. Wang, L. S. Li, D. Wang, T. Zhu, J. Xu, C. Yang, and Y. Li, Nature Photon. 1, 717-722 (2007).

[45] F. Qian, S. Gradecak, Y. Li, C-Y. Wen, and C. M. Lieber, Nano Lett. 5, 2287-2291 (2005).

[46] C. B. Murray, D. J. Norris, and M. G. Bawendi, J. Am. Chem. Soc. 115, 8706-8715 (1992).

[47] S. V. Gaponenko, Introduction to Nanophotonics, (Cambridge University Press, Cambridge, 2010).

[48] U. Woggon and S. V. Gaponenko, Phys. Status Solidi B 189, 285 (1995).

[49] A. M. Morales and C. M. Lieber, Science 279, 208-211 (1998).

[50] J. T. Hu, T. W. Odom, and C. M. Lieber, Acc. Chem. Res. 32, 435-455 (1999).

[51] J. T. Hu, L. S. Li, W. D. Yang, L. Manna, L. W. Wang, and A. P. Alivisatos, Science 292, 2060-2063 (2001).

[52] V. L. Colvin, M. C. Schlamp, and A. P. Alivisatos, Nature 370, 354-357 (1994).

[53] S. Coe, W.-K. Woo, M. Bawendi, and V. Bulovic, Nature 420, 800-803 (2002).

[54] W. U. Huynh, J. J. Dittmer, and A. P. Alivisatos, Science 295, 2425-2427 (2002).

[55] H. V. Demir, S. Nizamoglu, T. Erdem, E. Mutlugun, N. Gaponik, and A. Eychmüller, Nano Today 6, 632-647 (2011).

[56] X. Duan, Y. Huang, Y. Cui, J. Wang, and C. M. Lieber, Nature 409, 66-69 (2001).

[57] M. H. Huang, S. Mao, H. Feick, H. Yan, Y. Wu, H. Kid, E. Weber, R. Russo, and P. Yang, Science 292, 1897-1899 (2001).

[58] M. S. Gudiksen, L. J. Lauhon, J. Wang, D. C. Smith, and C. M. Lieber, Nature 415, 617-620 (2002).

[59] Y. Huang, X. Duan, and C. M. Lieber, Small 1, 142-147 (2005). 
[60] Y. Li, F. Qian, J. Xiang, and C. M. Lieber, Mater. Today 9 , 18-27 (2006).

[61] L. Novotny and B. Hecht, Principles of Nano-optics, (Cambridge, Cambridge, 2006).

[62] Z. Lin, H. Li, A. Franceschetti, and M. T. Lusk, ACS Nano 6, 4029-4038 (2012).

[63] S. A. Crooker, J. A. Hollingsworth, S. Tretiak, and V. I. Klimov, Phys. Rev. Lett. 89, 186802 (2002).

[64] S. W. Clark, J. M. Harbold, and F. W. Wise, J. Phys. Chem. C. 111, 7302-7305 (2007).

[65] S. F. Wuister, R. Koole, C. d. M. Donega, and A. Meijerink, J. Phys. Chem. B. 109, 5504-5508 (2005).

[66] S. Nizamoglu and H. V. Demir, J. Appl. Phys. Lett. 105, 083112 (2009).

[67] B. N. Pal, Y. Ghosh, S. Brovelli, R. Laocharoensuk, V. I. Klimov, J. A. Hollingsworth, and H. Htoon, Nano Lett. 12, 331-336 (2012).

[68] A. L. Rogach, T. A. Klar, J. M. Lupton, A. Meijerink, and J. Feldmann, J. Mater. Chem. 19, 1208-1221 (2009).

[69] T. Franzl, T. A. Klar, S. Schietinger, A. L. Rogach, and J. Feldmann, Nano Lett. 4, 1599-1603 (2004).

[70] T. A. Klar, T. Franzl, A. L. Rogach, and J. Feldmann, Adv. Mater 17, 769-773 (2005).

[71] M. Achermann, M. A. Petruska, S. A. Crooker, and V. I. Klimov, J. Phys. Chem. B. 107, 13782-13787 (2003).

[72] S. Nizamoglu and H. V. Demir, Opt. Exp. 16, 13961-13968 (2008).

[73] T. Franzl, A. Shavel, A. L. Rogach, N. Gaponik, T. A. Klar, A. Eychmüller, and J. Feldmann, Small 1, 392-395 (2005).

[74] S. Nizamoglu and H. V. Demir, Appl. Phys. Lett. 95, 151111 (2009).

[75] S. Nizamoglu, O. Akin, and H. V. Demir, Appl. Phys. Lett. 94, 243107 (2009).

[76] K. E. Knowles, M. T. Frederick, D. B. Tice, A. J. MorisCohen, and E. A. Weiss, J. Phys. Chem. Lett. 3, 18-26 (2012).

[77] G. D. Scholes and D. L. Andrews, Phys. Rev. B 72, 125331 (2005).

[78] A. O. Govorov, Phys. Rev. B 71, 155323 (2005).

[79] S. Y. Kruchinin, A. V. Fedorov, A. V. Baranov, T. S. Perova, and K. Berwick, Phys. Rev. B 78, 125311 (2008).

[80] A. Nazir, Phys. Rev. Lett. 103, 146404 (2009).

[81] C. Curutchet, A. Franceschetti, A. Zunger, and G. D. Scholes, J. Phys. Chem. C 112, 13336-13341 (2008).

[82] S. Halvini, A. Sitt, I. Hadar, and U. Banin, ACS Nano 6, 2758-2765 (2012).

[83] D. A. B. Miller, Opt. Photon. News 1, 7-15 (1990).

[84] M. J. Bowers II, J. R. McBride, and S. J. Rosenthal, J. Am. Chem. Soc. 127, 15378-15379 (2005).

[85] H.-S. Chen, D.-M. Yeh, C.-F. Lu, C.-F. Huang, W.-Y. Shiao, J.-J. Huang, C. C. Yang, I.-S. Liu, and W.-F. Su, IEEE Photon. Technol. Lett. 18, 1430-1432 (2006).

[86] S. Nizamoglu, T. Ozel, E. Sari, and H. V. Demir, Nanotechnology 18, 065709 (2007).

[87] S. Nizamoglu, G. Zengin, and H. V. Demir, Appl. Phys. Lett. 92, 031102 (2008).

[88] H. V. Demir, S. Nizamoglu, E. Mutlugun, T. Ozel, S. Sampra, N. Gaponik, and A. Eychmuller, Nanotechnology 19, 335203 (2008).
[89] S. Nizamoglu, E. Mutlugun, T. Ozel, H. V. Demir, S. Sapra, N. Gaponik, and A. Eychmüller, Appl. Phys. Lett. 92, 113110 (2008).

[90] C. Dang, J. Lee, Y. Zhang, J. Han, C. Breen, J. S. Steckel, S. Coe-Sullivan, and A. Nurmikko, Adv. Mater. doi: 10. 1002/adma. 201202354.

[91] M. Achermann, M. A. Petruska, S. Kos, D. L. Smith, D. D. Koleske, and V. I. Klimov, Nature 429, 642-646 (2004).

[92] D. Basko, G. C. La Rocca, F. A. Bassani, and V. M. Agranovich, Eur. Phys. J. B. 8, 353-362 (1999).

[93] S. Kos, M. Achermann, V. I. Klimov, and D. L. Smith, Phys. Rev. B 71, 205309 (2005).

[94] S. Blumstengel, S. Sadofev, C. Xu, J. Puls, and F. Henneberger, Phys. Rev. Lett. 97, 237401 (2006).

[95] G. Itskos, G. Heliotis, P. G. Lagoudakis, J. Lupton, N. P. Barradas, E. Alves, S. Pereira, I. M. Watson, M. D. Dawson, J. Feldmann, R. Murray, and D. D. C. Bradley, Phys. Rev. B 76, 035344 (2007).

[96] C. R. Belton, G. Itskos, G. Heliotis, P. N. Stavrinou, P. G. Lagoudakis, J. Lupton, S. Pereira, E. Gu, C. Griffin, B. Guilhabert, I. M. Watson, A. R. Mackintosh, R. A. Pethrick, J. Feldmann, R. Murray, M. D. Dawson, and D. D. C. Bradley, J. Phys. D. : Appl. Phys. 41, 094006 (2008).

[97] S. Chanyawadee, P. G. Lagoudakis, R. T. Harley, D. G. Lidzey, and M. Henini, Phys. Rev. B 77, 193402 (2008).

[98] Y. Gladush, C. Piermarocchi, and V. Agranovich, Phys. Rev. B 84, 205312 (2011).

[99] J. J. Rindermann, G. Pozina, B. Monemar, L. Hultman, H. Amano, and P. G. Lagoudakis, Phys. Rev. Lett. 107, 236805 (2011).

[100] S. Lu and A. Madhukar, Nano Lett. 7, 3443-3451 (2007).

[101] S. Nizamoglu, E. Sari, J.-H. Baek, I.-H. Lee, and H. V. Demir, Phys. Status Solidi RRL 4, 178-180 (2010).

[102] S. Chanyawadee, R. T. Harley, M. Henini, D. V. Talapin, and P. G. Lagoudakis, Phys. Rev. Lett. 102, 077402 (2009).

[103] S. Chanyawadee, P. G. Lagoudakis, R. T. Harley, M. D. B. Charlton, D. V. Talapin, H. W. Huang, and C.-H. Lin, Adv. Mater. 22, 602-606 (2010).

[104] S. Nizamoglu, B. Guzelturk, D.-W. Jeon, I.-H. Lee, and H. V. Demir, Appl. Phys. Lett. 98, 163108 (2011).

[105] F. Zhang, J. Liu, G. You, C. Zhang, S. E. Mohney, M. J. Park, J. S. Kwak, Y. Wang, D. D. Koleske, and J. Xu, Opt. Exp. 20, 333-339 (2012).

[106] B. Guzelturk, S. Nizamoglu, D.-W. Jeon, I.-H. Lee, and H. V. Demir, Strong nonradiative energy transfer from the nanopillars of quantum wells to quantum dots: Efficent excitonic color conversion for light emitting diodes, CLEO: Science and Innovations, CW1L (2012).

[107] S. Nizamoglu, P. L. Hernandez-Martinez, E. Mutlugun, D. U. Karatay, and H. V. Demir, Appl. Phys. Lett. 100, 241109 (2012).

[108] J. Lee, A. O. Govorov, and N. A. Kotov, Nano Lett. 5, 2063-2069 (2005).

[109] S. Lu and A. Madhukar, Nano Lett. 7, 3443-3451 (2007).

[110] S. Lu, Z. Lingley, T. Asano, D. Harris, T. Barwicz, S. Guha, and A. Madhukar, Nano Lett. 9, 4548-4552 (2009).

[111] A. Dorn, D. B. Strasfeld, D. K. Harris, H.-S. Han, and M. G. Bawendi, ACS Nano 5, 9028-9033 (2011).

[112] P. L. Hernandez-Martinez and A. O. Govorov, Phys. Rev. B 78, 035314 (2008). 
[113] J. Y. Kim and F. E. Osterloh, J. Am. Chem. Soc. 127, 10152-10153 (2005).

[114] L.-J. Tzeng, C.-L. Cheng, and Y.-F. Chen, Opt. Exp. 33, 569-571 (2008)

[115] J.-Y. Chang, T. G. Kim, and Y.-M. Sung, Nanotechnology 22, 425708 (2011).

[116] J. M. Haremza, M. A. Hahn, and T. D. Krauss, Nano Lett. 2, 1253-1258 (2002).

[117] X. Peng, J. Chen, J. A. Misewich, and S. S. Wong, Chem. Soc. Rev. 38, 1076-1098 (2009).

[118] M. Grzelczak, M. A. Correa-Duarte, V. SalgueirinoMaceira, M. Giersig, R. Diaz, and L. M. Liz-Marzan, Adv. Mater. 18, 415-420 (2006).

[119] V. Biju, T. Itoh, Y. Baba, and M. Ishikawa, J. Phys. Chem. B. 110, 26068-26074 (2006).

[120] E. Shafran, B. D. Mangum, and J. M. Gerton, Nano Lett. 10, 4049-4054 (2010).

[121] B. Zebli, H. A. Vieyra, I. Carmeli, A. Hartschuh, J. P. Kotthaus, and A. W. Holleitner, Phys. Rev. B 79, 205402 (2009).

[122] E. Morales-Navarez, B. Perez-Lopez, L. B. Pires, and A. Merkoçi, Carbon 50, 2987-2993 (2012).

[123] S. Jander, A. Kornowski, and H. Weller, Nano Lett. 11, 5179-5183 (2011).

[124] I. L. Medintz, H. T. Uyeda, E. R. Goldman, and H. Mattoussi, Nature Mater. 4, 435-446 (2004).

[125] C. Dang, J. Lee, C. Breen, J. S. Steckel, S. Coe-Sullivan, and A. Nurmikko, Nature Nanotech. 7, 335-339 (2012).

[126] S. K. Saikin, A. Eisfeld, S. Valleau, and A. Aspuru-Guzik, Nanophotonics 2, 21-38 (2013).

[127] N. Tessler, V. Medvedev, M. Kazes, S. H. Kan, and U. Banin, Science 295, 1056-1058 (2002).

[128] M. Anni, L. Manna, R. Cingolani, D. Valerini, A. Creti, and M. Lomascolo, Appl. Phys. Lett. 85, 4169-4171 (2004).

[129] T.-W. F. Chang, S. Musikhin, L. Bakueva, L. Levina, M. A. Hines, P. W. Cyr, and E. H. Sargent, Appl. Phys. Lett. 84, 4295-4297 (2004).

[130] J. H. Warner, A. R. Watt, E. Thomsen, N. Heckenberg, P. Meredith, and H. Rubinsztein-Dunlop, J. Phys. Chem. B 109, 9001-9005 (2005).

[131] S.-K. Hong, Physica E 28, 66-75 (2005).

[132] M. Y. Odoi, N. I. Hammer, K. Sill, T. Emrick, and M. D. Barnes, J. Am. Chem. Soc. 128, 3506-3507 (2006).

[133] S. Kaufmann, T. Stöferle, N. Moll, R. F. Mahrt, U. Scherf, A. Tsami, D. V. Talapin, and C. B. Murray, Appl. Phys. Lett. 90, 071108 (2007).

[134] P. T. K. Chin, R. A. M. Hikmet, and R. J. Janssen, J. Appl. Phys. Lett. 104, 013108 (2008).

[135] T. Stöferle, U. Scherf, and R. F. Mahrt, Nano Lett. 9, 453456 (2009).

[136] A. Lutich, G. Jiang, A. S. Susha, A. L. Rogach, F. D. Stefani, and J. Feldmann, Nano Lett. 9, 2636-2640 (2009).

[137] A. Lutich, A. Pöschl, G. Jiang, F. D. Stefani, A. S. Susha, A. L. Rogach, and J. Feldmann, Appl. Phys. Lett. 96, 083109 (2010).

[138] E. Holder, N. Tessler, and A. L. Rogach, J. Mater. Chem. 18, 1064-1078 (2007).

[139] N. Tomczak, D. Janczewski, M. Han, and G. J. Vancso, Prog. Polym. Sci. 34, 393-430 (2009).
[140] P. Reiss, E. Couderc, J. D. Girolamo, and A. Pron, Nanoscale 3, 446-489 (2011).

[141] P. O. Anikeeva, J. E. Halpert, M. G. Bawendi, and V. Bulovic, Nano Lett. 7, 2196-2200 (2007).

[142] P. O. Anikeeva, J. E. Halpert, M. G. Bawendi, and V. Bulovic, Nano Lett. 7, 2532-2536 (2009).

[143] P. O. Anikeeva, C. F. Madigan, J. E. Halpert, M. G. Bawendi, and V. Bulovic, Phys. Rev. B 78, 085434 (2008).

[144] P. Jing, X. Yuan, W. Ji, M. Ikezawa, Y. A. Wang, X. Liu, L. Zhang, J. Zhao, and Y. Masumoto, J. Phys. Chem. C 114, 19256-19262 (2010).

[145] P. Jing, X. Yuan, W. Ji, M. Ikezawa, X. Liu, L. Zhang, J. Zhao, and Y. Masumoto, Appl. Phys. Lett. 99, 093106 (2011).

[146] P. O. Anikeeva, C. F. Madigan, S. A. Coe-Sullivan, J. S. Steckel, M. G. Bawendi, and V. Bulovic, Chem. Phys. Lett. 424, 120-125 (2006).

[147] G. Cheng, M. Mazzeo, A. Rizzo, Y. Li, Y. Duan, and G. Gigli, Appl. Phys. Lett. 94, 243506 (2009).

[148] Y. Q. Zhang and X. A. Cao, Appl. Phys. Lett. 97, 235115 (2010).

[149] G. Cheng, W. Lu, Y. Chen, and C.-M. Che, Opt. Exp. 37, 1109-1111 (2012).

[150] D. M. Willard, L. L. Carillo, J. Jung, and A. V. Orden, Nano Lett. 1, 469-474 (2001).

[151] A. R. Clapp, I. L. Medintz, J. M. Mauro, B. R. Fisher, M. G. Bawendi, and H. Mattoussi, J. Am. Chem. Soc. 126, 301-310 (2004).

[152] I. L. Medintz, A. R. Clapp, H. Mattoussi, E. R. Goldman, B. Fisher, and J. M. Mauro, Nat. Mater. 2, 630-638 (2003).

[153] I. L. Medintz, J. H. Konnert, A. R. Clapp, I. Stanish, M. E. Twigg, H. Mattoussi, J. M. Mauro, and J. R. Deschamps, Proc. Natl. Acad. Sci. USA 101, 9612-9617 (2004).

[154] I. L. Medintz and H. Mattoussi, Phys. Chem. Chem. Phys. 11, 17-45 (2009).

[155] W. R. Algar, D. Wegner, A. L. Huston, J. B. Blanco-Canosa, M. H. Stewart, A. Armstrong, P. E. Dawson, N. Hildebrandt, and I. L. Medintz, J. Am. Chem. Soc. 134, 1876-1891 (2012).

[156] M.-K. So, C. Xu, A. M. Loening, S. S. Gambhir, and J. Rao, Nature Biotech. 24, 339-343 (2006).

[157] H. Yao, Y. Zhang, F. Xiao, Z. Xia, and J. Rao, Angew. Chem. Int. Ed. 46, 4346-4349 (2007).

[158] X. Huang, L. Li, H. Qian, C. Dong, and J. Ren, Angew. Chem. 118, 5264-5267 (2006).

[159] M.-S. Wu, H.-W. Shi, J.-J. Xu, and H.-Y. Chen, Chem. Commun. 47, 7752-7754 (2011).

[160] L. Li, M. Li, Y. Sun, J. Li, L. Sun, G. Zou, X. Zhang, and W. Jin, Chem. Commun. 47, 8292-9294 (2011).

[161] M. Artemyev, E. Ustinovich, and I. Nabiev, J. Am. Chem. Soc. 131, 8061-8065 (2009).

[162] E. Mutlugun, S. Nizamoglu, and H. V. Demir, Appl. Phys Lett. 95, 033106 (2009).

[163] S. Sarkar, R. Bose, S. Jana, N. R. Jana, and N. Pradhan, J. Phys. Chem. Lett. 1, 636-640 (2010).

[164] M. Achermann, S. Jeong, L. Balet, G. A. Montano, and J. A. Hollingsworth, ACS Nano 5, 1761-1768 (2011).

[165] S. Halivni, A. Sitt, I. Hadar, and U. Banin, ACS Nano 6, 2758-2765 (2012)

[166] T. Takagahara, Phys. Rev. B 47, 4569-4584 (1993). 
[167] T. Someya, H. Akiyama, and H. Sakaki, Phys. Rev. Lett. 76, 2965-2968 (1996).

[168] E. A. Muljarov, E. A. Zhukov, V. S. Dneprovskii, and Y. Masumoto, Phys. Rev. B 62, 7420-7432 (2000).

[169] Q. Li, X. Gong, C. Wang, J. Wang, K. Ip, and S. Hark, Adv. Mater. 16, 1436-1440 (2004).

[170] R. Chen, D. Li, B. Liu, Z. Peng, G. G. Gurzadyan, Q. H. Xiong, and H. Sun, Nano Lett. 10, 4956-4961 (2010).

[171] M. H. Huang, S. Mao, H. Feick, H. Yan, Y. Wu, H. Kind, E. Weber, R. Russo, and P. Yang, Science 292, 1897-1899 (2001).

[172] R. Agarwal, C. J. Barrelet, and C. M. Lieber, Nano Lett. 5, 917-920 (2005).

[173] R. Chen, M. I. B. Utama, Z. Peng, B. Peng, Q. H. Xiong, and H. Sun, Adv. Mater. 23, 1404-1408 (2011).

[174] X. Xu, Y. Zhao, E. J. Sie, Y. Lu, B. Liu, S. A. Ekahana, X. Ju, Q. Jiang, J. Wang, H. Sun, T. C. Sum, C. H. A. Huan, Y. P. Feng, and Q. H. Xiong, ACS Nano 5, 3660-3669 (2011).

[175] L. J. Lauhon, M. S. Gudiksen, and C. M. Lieber, Philos. Trans. R. Soc. Lond. A 362, 1247-1260 (2004).

[176] F. Qian, Y. Li, S. Gradecak, H.-G. Park, Y. Dong, Y. Ding, Z. L. Wang, and C. M. Lieber, Nature Mater. 7, 701-706 (2008).

[177] F. Qian, M. Brewster, S. K. Lim, Y. Ling, C. Greene, O. Laboutin, J. W. Johnson, S. Gradecak, Y. Cao, and Y. Li, Nano Lett. 12, 3344-3350 (2012).

[178] R. Könenkamp, R. C. Word, and C. Schlegel, Appl. Phys. Lett. 85, 7004-6006 (2004).

[179] R. Könenkamp, R. C. Word, and M. Godinez, Nano Lett. 5, 2005-2008 (2005).

[180] J. Bao, M. A. Zimmler, and F. Capasso, Nano Lett. 6, 17191722 (2006)

[181] A. Nadarajah, R. C. Word, J. Meiss, and R. Könenkamp, Nano Lett. 8, 534-537 (2008).

[182] X.-M. Zhang, M.-Y. Lu, Y. Zhang, L.-J. Chen, and Z. L. Wang, Adv. Mater. 21, 2767-2770 (2009).

[183] O. Lupan, T. Pauporte, and B. Viana, Adv. Mater. 22, 3298 3302 (2010).

[184] M. A. Zimmler, T. Voss, C. Ronning, and F. Capasso, Appl. Phys. Lett. 94, 241120 (2009).

[185] B. Yan, R. Chen, W. Zhou, J. Zhang, H. Sun, H. Gong, and T. Yu, Nanotechnology 21, 445706 (2010).

[186] Q. Yang, W. Wang, S. Xu, and Z. L. Wang, Nano Lett. 11, 4012-4017 (2011).

[187] S. Nanot, E. H. Haroz, J.-H. Kim, R. H. Hauge, and J. Kono, Adv. Mater. 24, 4977-4994 (2012).

[188] C. D. Spataru, S. Ismail-Beigi, L. X. Benedict, and S. G. Louie, Phys. Rev. Lett. 92, 077402 (2004).

[189] V. Perebeinos, J. Tersoff, P. Avouris, Phys. Rev. Lett. 92, 257402 (2004).

[190] F. Wang, G. Dukovic, L. E. Brus, and T. F. Heinz, Science 308, 838-843 (2005).

[191] I. V. Bondarev, L. M. Woods, and K. Tatur, Phys. Rev. B 80, 085407 (2009).

[192] A. J. Bindl, M.-Y. Wu, F. C. Prehn, and M. S. Arnold, Nano Lett. 11, 455-460 (2010).

[193] A. J. Siitonen, D. A. Tsyboulski, S. M. Bachilo, and R. B. Weisman, Nano Lett. 10, 1595-1599 (2010).

[194] S. Wang, M. Kahfizov, X. Tu, M. Zheng, and T. D. Krauss, Nano Lett. 10, 2381-2386 (2010).
[195] A. Högele, C. Galland, M. Winger, and A. Imamoglu, Phys. Rev. Lett. 100, 217401 (2008).

[196] T. Mueller, M. Kinoshita, M. Steiner, V. Perebeinos, A. A. Bol, D. B. Farmer, and P. Avouris, Nature Nanotech. 5, 27-31 (2009).

[197] S. Wang, Q. Zheng, L. Yang, Z. Zhang, Z. Wang, T. Pei, L. Ding, X. Liang, M. Gao, Y. Li, and L.-M. Peng, Nano Lett. 11, 23-29 (2011).

[198] X. Xie, A. E. Islam, M. A. Wahab, L. Ye, X. Ho, M. A. Alam, and J. A. Rogers, ACS Nano doi:10. 1021/nn3025496 (2012).

[199] P. H. Tan, A. G. Rozhin, T. Hasan, P. Hu, V. Scardaci, W. I. Milne, and A. C. Ferrari, Phys. Rev. Lett. 99, 137402 (2007).

[200] H. Qian, C. Georgi, N. Anderson, A. A. Green, M. C. Hersam, L. Novotny, and A. Hartschuh, Nano Lett. 8, 13631367 (2008).

[201] H. Qian, C. Georgi, N. Anderson, A. A. Green, M. C. Hersam, L. Novotny, and A. Hartschuh, Phys. Status Solidi B 245, 2243-2246 (2008).

[202] J. Lefebvre and P. Finnie, J. Phys. Chem. C 113, 7536-7540 (2009).

[203] H. Hirori, K. Matsuda, and Y. Kanemitsu, Phys. Rev. B 78, 113409 (2008).

[204] T. Koyama, Y. Asada, N. Hikosaka, Y. Miyata, H. Shinohara, and A. Nakamura, ACS Nano 5, 5881-5887 (2011).

[205] A. O. Govorov, G. W. Bryant, W. Zhang, T. Skeini, J. Lee, N. A. Kotov, J. M. Slocik, and R. R. Naik, Nano Lett. 6, 984-994 (2006).

[206] N. T. Fofang, T.-H. Park, O. Neumann, N. A. Mirin, P. Nordlander, and N. J. Halas, Nano Lett. 8, 3481-3487 (2008).

[207] P. D. Artuso and G. W. Bryant, Nano Lett. 8, 2106-2111 (2008).

[208] D. E. Gomez, K. C. Vernon, P. Mulvaney, and T. J. Davis, Nano Lett. 10, 274-278 (2010).

[209] A. Manjavacas, F. J. G. d. Abajo, and P. Nordlander, Nano Lett. 11, 2318-2323 (2011).

[210] O. Kulakovich, N. Strekal, A. Yaroshevich, S. Maskevich, S. Gaponenko, I. Nabiev, U. Woggon, and M. Artemyev, Nano Lett. 2, 1449-1452 (2002).

[211] T. E. Jennings, J. C. Schlatterer, M. P. Singh, N. L. Greenbaum, and G. F. Strouse, Nano Lett. 6, 1218-1324 (2006).

[212] O. G. Tovmachenko, C. Graf, D. J. van den Heuvel, A. van Blaaderen, and H. C. Gerritsen, Adv. Mater. 18, 91-95 (2006).

[213] T. Pons, I. L. Medintz, K. E. Sapsford, S. Higashiya, A. F. Grimes, D. S. English, and H. Matoussi, Nano Lett. 7, 3157-3164 (2007).

[214] B. Peng, Q. Zhang, X. Liu, Y. Li, H. V. Demir, C. H. A. Huan, T. Z. Sum, and Q. H. Xiong, ACS Nano 6, 6250-6259 (2012).

[215] J. Lee, A. O. Govorov, J. Dulka, and N. A. Kotov, Nano Lett. 4, 2323 (2004)

[216] J. Lee, T. Javed, T. Skeini, A. O. Govorov, G. W. Bryant, and N. A. Kotov, Angew. Chem. 45, 4819 (2006). 
[217] A. O. Govorov, J. Lee, and N. A. Kotov, Phys. Rev. B 76, 125308 (2007).

[218] V. Faessler, C. Hrelescu, A. A. Lutich, L. Osinkina, S. Mayilo, F. Jackel, and J. Feldmann, Chem. Phys. Lett. 508, 67-70 (2011).

[219] M. Lunz, V. A. Gerard, Y. K. Gunko, V. Lesnyak, N. Gaponik, A. S. Susha, A. L. Rogach, and A. L. Bradley, Nano Lett. 11, 3341-3345 (2011).
[220] T. Ozel, S. Nizamoglu, M. A. Sefunc, O. Samarskaya, I. O. Ozel, E. Mutlugun, V. Lesnyak, N. Gaponik, A. Eychmuller, S. V. Gaponenko, and H. V. Demir, ACS Nano 5, 1328-1334 (2011).

[221] P. Andrew and W. L. Barnes, Science 290, 785-788 (2000).

[222] P. Andrew and W. L. Barnes, Science 306, 1002-1005 (2004).

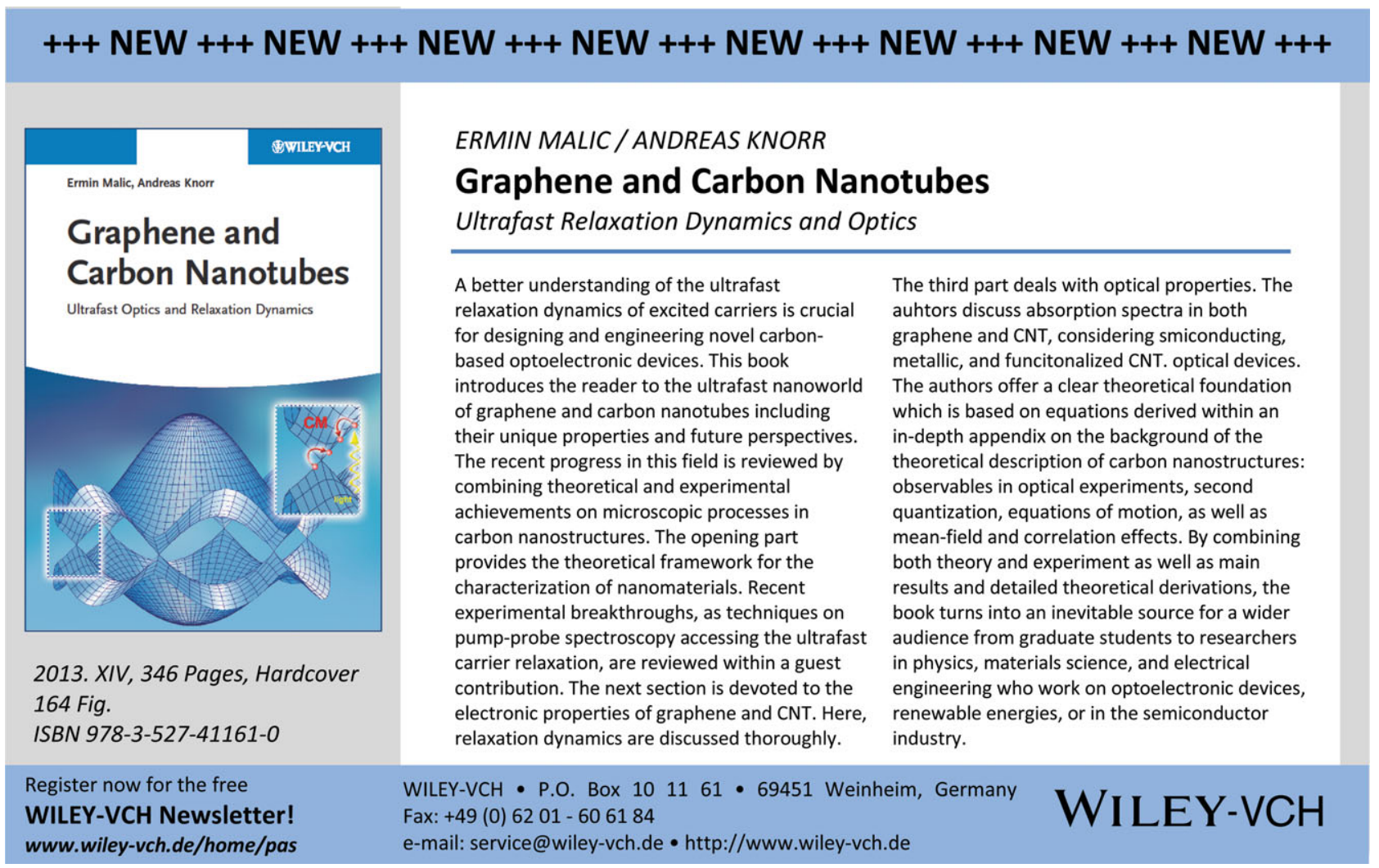

